A\&A 630, A112 (2019)

https://doi.org/10.1051/0004-6361/201935968

(c) B. Mancillas et al. 2019

\title{
Molecular gas content of shell galaxies ${ }^{\star}$
}

\author{
B. Mancillas ${ }^{1}$, F. Combes ${ }^{1,2}$, and P.-A. Duc ${ }^{3}$ \\ 1 Observatoire de Paris, LERMA, PSL University, CNRS, Sorbonne University, UPMC, Paris, France \\ e-mail: brisa.mancillas@obspm.fr \\ 2 Collège de France, 11 place Marcelin Berthelot, 75005 Paris, France \\ 3 Université de Strasbourg, CNRS, Observatoire de Strasbourg, 67000 Strasbourg, France
}

Received 27 May 2019 / Accepted 22 August 2019

\begin{abstract}
Shells are fine stellar structures that are identified by their arc-like shapes around a galaxy. They are currently thought to be vestiges of galaxy interactions and/or mergers. The study of their number, geometry, stellar populations, and gas content can help us to derive the interaction or merger history of a galaxy. Numerical simulations have proposed a mechanism of shell formation through phase wrapping during a radial minor merger. Alternatively, there could be merely a space wrapping, when particles have not yet radially oscillated, but are bound by their radial expansion, or produce an edge-brightened feature. These can be distinguished because they are expected to keep a high radial velocity. While shells are first a stellar phenomenon, HI and CO observations have revealed neutral gas associated with shells. Some of the gas, the most diffuse and dissipative, is expected to be quickly driven to the center if it is traveling on nearly radial orbits. Molecular gas, which is distributed in dense clumps, is less dissipative, and may be associated with shells. It can then determine the shell velocity, which is too difficult to obtain from stars. We present here a search for molecular gas in nine shell galaxies with the IRAM-30 m telescope. Six of them are detected in their galaxy center, and in three galaxies, we clearly detect molecular gas in shells. The derived amount of molecular gas varies from $1.5 \times 10^{8}$ to $3.4 \times 10^{9} M_{\odot}$ in the shells. For two of them (Arp 10 and NGC 3656), the shells are characteristic of an oblate system. Their velocity is nearly systemic, and we conclude that these shells are phase wrapped. In the third galaxy (NGC 3934), the shells appear to participate in the rotation. Follow-up with higher spatial resolution is required to conclude.
\end{abstract}

Key words. galaxies: interactions - galaxies: ISM - galaxies: kinematics and dynamics - galaxies: peculiar - galaxies: structure

\section{Introduction}

It is well accepted that galaxy interactions frequently lead to merging, and that it must have played a fundamental role in the formation and evolution of galaxies (Toomre \& Toomre 1972). Most of our understanding of galaxy interactions has been obtained through numerical simulations (see for a review Barnes \& Hernquist 1992). Among the best vestiges of galaxy interactions are shells and ripples (Schweizer \& Seitzer 1992), which are often present around merger remnants. Shells, which are composed of stars, were originally observed around elliptical and S0 galaxies, which are poor-gas systems (Carter et al. 1982). A convincing theory has been developed to account for the formation of shells around early-type galaxies as a phasewrapping phenomenon (Quinn 1984; Dupraz \& Combes 1986 , 1987; Hernquist \& Quinn 1989). According to the theory, the stars modeled as collisionless particles, which were originally bound to the merging companions, are liberated and oscillate with different periods of oscillation around the primary galaxy. They accumulate near the apocenters of their orbits to form shelllike features. This phenomenon is also predicted to occur in the last phase of any merging between two spiral galaxies. The endproduct appears to be an elliptical, with a de Vaucouleurs law as light profile (e.g., Schweizer 1990). The late infall of tidal tails and debris, predominantly consisting of stars, initiates the phase wrapping and subsequent shell formation. Most spiral merger

* Based on observations carried out with the $30 \mathrm{~m}$ telescope of Institute of RAdioastronomy in Millimeter (IRAM). remnants, such as the prototypical NGC 7252, appear indeed to possess shells (Schweizer 1990).

The morphology of shells may help to reveal the 3D structure of the galaxy potential: if the galaxy is prolate, shells align along the major axis and are interleaved in radius, while the shells are found randomly in azimuth near the equatorial plane of an oblate galaxy (Dupraz \& Combes 1986). All these are phasewrapped shells, where stars are accumulating at the apocenter of their oscillations. However, as Hernquist \& Quinn (1988) have pointed out, there could also be some features such as tails or streams, edge-brightened or only space-bounded, that do not fall under phase wrapping, but only under space wrapping.

This space-wrapping scenario was invoked when neutral hydrogen was observed in systems containing shells. Schiminovich et al. (1994, 1995) have detected diffuse HI gas associated with the stellar shells (Centaurus A and NGC 2865). These observations were a priori surprising in the phase-wrapped scenario because we believe that the diffuse gaseous and stellar components do not have the same behavior when they approach the center of the potential well in quasi-radial orbits (Weil \& Hernquist 1993). The gas was expected to infall to the center, without being able to oscillate back. However, part of the gas could be structured in clouds and be less dissipative, and the orbits might also be less radial. Simulating a minor merger with clumpy gas, Combes \& Charmandaris (2000) have shown that indeed, a large fraction of the gas could follow the stars in phasewrapped shells. Because the gas in the outer parts of the merging companion is less strongly bound than the stars, it is liberated first and is less slowed down by dynamical friction: gaseous 
shells have a slightly larger radius than stellar shells. In Centaurus $\mathrm{A}$, the $\mathrm{HI}$ is associated with the outermost faint stellar shells. It is displaced to the outside of the optical shells, and has a high rotation velocity, suggesting that only a companion with a more circular orbit is able to produce this HI ring (Schiminovich et al. 1994). Other cases of HI shells displaced with respect to the stellar shells have been reported (Schiminovich et al. 1997; van Gorkom 2000).

Detecting gas in shells is useful to be able to determine their velocity. Obtaining a stellar velocity is costly and requires the use of spectrographs with IFU capabilities on large telescopes. The HI maps suffer from low spatial resolution, therefore molecular gas might be one of the best ways to determine shell kinematics. The latter is important to try distinguishing between the different scenarios: in the phase-wrapping model, stars have zero radial velocity in the shells, which correspond to their apocenter Velocities might be much higher in the space-wrapping model. In order to obtain more insight into the process of gaseous shell formation and into the behavior of gas in shell galaxies in general, we performed observations of $\mathrm{CO}(1-0)$ and $\mathrm{CO}(2-1)$ emission lines in the shell regions of eight early-type galaxies, two of them taken from the CO survey of ATLAS ${ }^{3 \mathrm{D}}$ sample (Young et al. 2011), and in five of them, $\mathrm{H}_{2}$ gas is detected in their center. In all cases, we re-observed the center positions to obtain a better signal-to-noise ratio $(\mathrm{S} / \mathrm{N})$. We detected $\mathrm{CO}$ emission associated with shells in three galaxies and detected molecular gas in six galaxies, where we made small maps. Out of the nine systems we observed, only two were not detected at all (NGC 5018 and NGC 7600). NGC 474 is not detected in its center, but $\mathrm{CO}$ is present in its companion. We then examine the expected association between the stellar and gaseous (HI or CO) shells, and discuss the various scenarios, taking the predictions from N-body numerical simulations into account.

The observations are described in Sect. 2, the sample of shell galaxies in Sect. 3, and the results are presented in Sect. 4. Finally, the interpretation and discussion are gathered in Sect. 5, and the conclusions in Sect. 6.

\section{Observations and data reduction}

Observations of the ${ }^{12} \mathrm{CO}(1-0)$ and ${ }^{12} \mathrm{CO}(2-1)$ emission have been carried out at the IRAM 30-m telescope at Pico Veleta, Granada, Spain, during 2017 July (for Arp 230, NGC 474, and NGC 3934), then September, October, and 2000 November (for the remaining sources). The beam full width at half-maximum (FWHM) was $23^{\prime \prime}$ and $11^{\prime \prime}$ at the frequencies of $115 \mathrm{GHz}$ and $230 \mathrm{GHz}$, respectively. The SIS receivers (EMIR) were used for observations in the wobbler-switching mode, with reference positions offset by \pm 120 arcsec in azimuth. The main-beam efficiency of IRAM is $\eta_{\mathrm{mb}}=T_{A}^{*} / T_{\mathrm{mb}}=0.82$ and 0.64 at $115 \mathrm{GHz}$ and $230 \mathrm{GHz}$, respectively. The system temperatures ranged between $190 \mathrm{~K}$ and $420 \mathrm{~K}$ at $2.6 \mathrm{~mm}$ and between $240 \mathrm{~K}$ and $600 \mathrm{~K}$ at $1.3 \mathrm{~mm}$. The pointing was regularly checked every $2 \mathrm{~h}$ on a nearby planet or a bright continuum source, and the focus was reviewed after each sunrise or if a suitable planet was available, as well as at the beginning of each night. The time on source typically ranged from $30 \mathrm{~min}$ to $1 \mathrm{~h}$; this depended on the weather. Two backends were used simultaneously, the autocorrelator WILMA, and the Fourier transform spectrometer FTS. The rms noise level was $\sigma_{\mathrm{mb}} \sim 1.5 \mathrm{mK}\left[T_{A}^{*}\right]$ with a spectrometer resolution of $40 \mathrm{~km} \mathrm{~s}^{-1}$ for $114 \mathrm{GHz}$ and $\sigma_{\mathrm{mb}} \sim 3.0 \mathrm{mK}\left[T_{A}^{*}\right]$ with a spectrometer resolution of $20 \mathrm{~km} \mathrm{~s}^{-1}$ for $230 \mathrm{GHz}$. The various pointings observed on the shell galaxies are indicated in Figs. 1-8.

\section{Sample}

We have selected shell galaxies that can be observed from the northern hemisphere, which were previously observed in HI$21 \mathrm{~cm}$ and in CO in the millimeter domain (see Table 1). In general, they are quite rich in neutral gas, except for NGC 7600. The properties of the nine shell galaxies selected in our sample are gathered in Table 1. The $\mathrm{H}_{2}$ masses in this table are those that were known before our study, and all luminosities and masses were scaled to the adopted distances. References in this table are for $\mathrm{HI}$ and $\mathrm{H}_{2}$. We list the detailed properties of each system below.

\section{1. $\operatorname{Arp} 230$}

Arp (1966) was the first to classify the galaxy as peculiar because of its faint stellar arcs, which were not yet called shells. Arp 230 (IC51) is now considered to be one of the prototypes for shell galaxies that have formed around a prolate object, with shells phase wrapped from the radial infall of a small satellite (Quinn 1984). The geometry of the shells is characteristic (see Fig. 1), they are aligned with the major axis, and interleaved in their distance to the center (Dupraz \& Combes 1986). At least eight shells can be counted, the most distant lies at $11 \mathrm{kpc}$, which is rather rare, although not as complex as the situation in NGC 3923 (Prieur 1988). As is frequently the case in shell systems, the first shell of Arp 230 (i.e., the most distant) is connected to the primary galaxy by a faint distorted tail or plume.

An HI mass of $1.59 \times 10^{9} M_{\odot}$ has been detected in this merged system with the Green Bank 140foot telescope (Richter et al. 1994). Schiminovich et al. (2013) mapped the HI in Arp 230 with the Very Large Array (VLA) and a resolution of $\sim 0.8 \mathrm{kpc}$. The HI is not aligned with the stellar shells, but perpendicular to the major axis, like a polar ring, aligned with the dust lane. The total HI mass detected with the VLA is $1.1 \times 10^{9} M_{\odot}$. McGaugh $\&$ Bothun (1990) together with Prieur (1988) found that the color of the shells is bluer than the main body of the Arp 230 galaxy, suggesting that the merger provided gas and star formation to the shells.

Galletta et al. (1997) have detected $\mathrm{CO}(1-0)$ in the center with the Kitt Peak $12 \mathrm{~m}$ telescope (beam of $1 \mathrm{arcmin}$ ), and derived $M\left(\mathrm{H}_{2}\right)=8.9 \times 10^{8} M_{\odot}$. The central dust lane corresponds to a molecular disk in rotation in the center, mapped in $\mathrm{CO}(1-$ $0)$ with ALMA at $\sim 0.5 \mathrm{kpc}$ resolution (Ueda et al. 2014). The corresponding $\mathrm{H}_{2}$ mass is $1.7 \times 10^{8} M_{\odot}$.

\subsection{NGC $474 / 470(\operatorname{Arp} 227)$}

The group has been observed in the optical by Rampazzo et al. (2006, 2007), and with high sensitivity by Duc et al. (2015). In the latter CFH-Megacam image, NGC 474 appears as a spectacular shell system, where shells are distributed randomly at all azimuth (see Fig. 2). This geometry corresponds to stellar shells that are aligned in the equatorial band of an oblate galaxy (Dupraz \& Combes 1986). Superposed on the shell systems is a radial tail or plume that stems from the past merger.

The first HI observations did not separate the two galaxies of the pair (Huchtmeier 1982). Interferometric observations with the VLA have shown that most of the HI reservoir belongs to the spiral NGC 470, but a long tail and bridge cover NGC 474, and therefore the early-type shell galaxy may possess some interstellar gas (Rampazzo et al. 2006), see also Fig. 3.

As early as 1993, Sofue et al. (1993) detected CO(1-0) emission toward Arp 227A (NGC 470), the late-type galaxy of the 
Table 1. Properties of the nine shell galaxies.

\begin{tabular}{|c|c|c|c|c|c|c|c|c|c|}
\hline Name & $\begin{array}{c}\text { RA } \\
\text { J2000 }\end{array}$ & $\begin{array}{c}\text { Dec } \\
\text { J2000 }\end{array}$ & $\begin{array}{c}c z \\
\mathrm{~km} \mathrm{~s}^{-1}\end{array}$ & $\begin{array}{c}D \\
\mathrm{Mpc}\end{array}$ & $\begin{array}{c}M(\mathrm{HI}) \\
\log M_{\odot}\end{array}$ & $\begin{array}{c}L(\mathrm{IR}) \\
\log L_{\odot}\end{array}$ & $\begin{array}{l}M\left(\mathrm{H}_{2}\right) \\
\log M_{\odot}\end{array}$ & Ref. & $\begin{array}{l}\text { Type } \\
\text { NED }\end{array}$ \\
\hline Arp 230 & $00: 46: 24.2$ & $-13: 26: 32$ & 1720 & 24.2 & 9.20 & 9.49 & 8.95 & (1) & SOpec \\
\hline NGC 474 & 01:20:06.7 & $+03: 24: 55$ & 2315 & 32.2 & 9.63 & 8.03 & $<7.7$ & (2) & SA0 \\
\hline Arp 10 & $02: 18: 26.3$ & $+05: 39: 14$ & 9108 & 120.0 & 10.2 & 10.5 & - & (3) & S? \\
\hline NGC 3032 & 09:52:08.1 & $+29: 14: 10$ & 1562 & 21.2 & 8.15 & 9.34 & 8.42 & (4) & $\mathrm{SABO}$ \\
\hline NGC 3656 & $11: 23: 38.6$ & $+53: 50: 32$ & 2870 & 42.6 & 9.27 & 9.99 & 9.67 & (5) & IOpec \\
\hline NGC 3934 & $11: 52: 12.5$ & $+16: 51: 05$ & 3779 & 49.2 & 9.51 & 9.98 & 9.32 & (6) & S? \\
\hline NGC 5018 & 13:13:01.0 & $-19: 31: 05$ & 2816 & 37.5 & 8.81 & 9.54 & $<7.4$ & (7) & E3 \\
\hline NGC 7600 & $23: 18: 53.8$ & $-07: 34: 50$ & 3483 & 39.0 & $<7.5$ & - & - & (8) & S0 \\
\hline Arp $295 N$ & $23: 42: 00.8$ & $-03: 36: 55$ & 6966 & 93.0 & 10.2 & 10.9 & 10.2 & (9) & Sbpec \\
\hline
\end{tabular}

Notes. All $\mathrm{H}_{2}$ data are centered and are representative of the total molecular content. $L($ IR) data are from the NED.

References. (1) Schiminovich et al. (2013) for HI, Ueda et al. (2014) for CO - (2) Rampazzo et al. (2006) for HI, Combes et al. (2007) for CO (3) Charmandaris \& Appleton (1996) for HI - (4) Lucero \& Young (2013) for HI, Combes et al. (2007) for CO - (5) Balcells et al. (2001) for HI, Young (2002) for CO - (6) Galletta et al. (1997) for HI and CO - (7) Kim et al. (1988) for HI, Ueda et al. (2014) for CO - (8) Serra et al. (2008) for HI - (9) Hibbard \& van Gorkom (1996) for HI and CO.

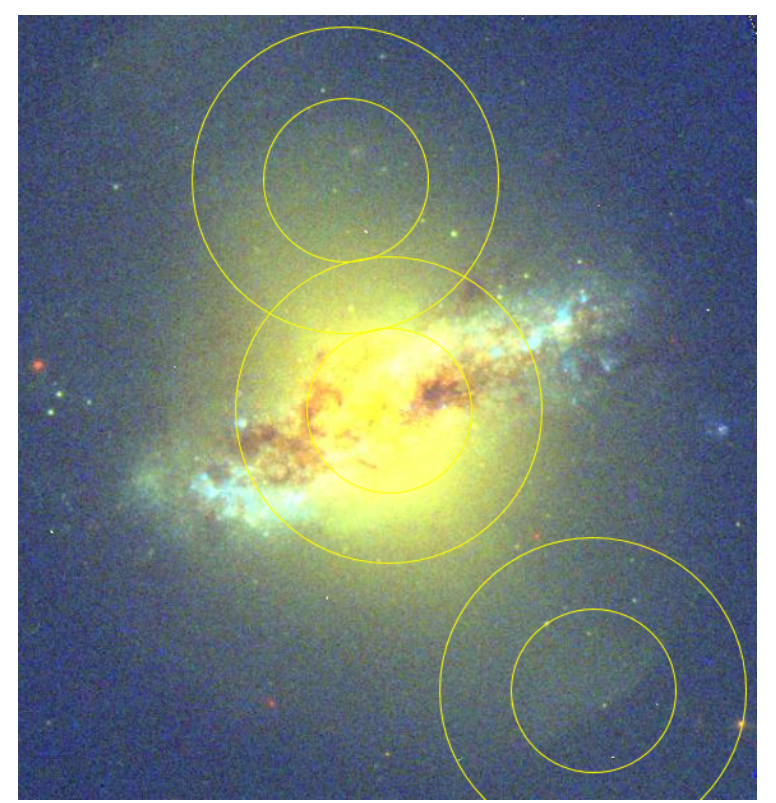

Fig. 1. $\mathrm{CO}(1-0) 23^{\prime \prime}$ and $\mathrm{CO}(2-1) 11^{\prime \prime}$ beams observed toward Arp 230 are overlaid on an HST composite image (F336W, F555W, and F814W). The two offset positions (NE and SW) are centered on shells.

pair, and derived an $\mathrm{H}_{2}$ mass of $2.3 \times 10^{8} M_{\odot}$. However, toward the shell early-type galaxy NGC 474, Combes et al. (2007) found an upper limit of $M\left(\mathrm{H}_{2}\right)<3.8 \times 10^{7} M_{\odot}$, with the IRAM$30 \mathrm{~m}$ telescope. We here obtain a lower upper limit for the CO emission toward the center, and then did not point toward the shells because the emission there is expected to be even lower. We observed the companion NGC 470 to determine how much available gas mass existed there that could have fallen towards NGC 474.

Lim et al. (2017) studied the globular cluster (GC) population in the NGC 474 galaxy. GCs and their color and age are good tracers of the merging history of a system. The violent starbursts triggered by the major mergers of gas-rich spirals favor the formation of metal-rich and red GC (Whitmore et al. 1993; Chien et al. 2007; Renaud et al. 2015), while the dry minor merger of small-mass satellites brings relatively blue GCs into the halo of a massive primary. In the outer parts of NGC 474, the

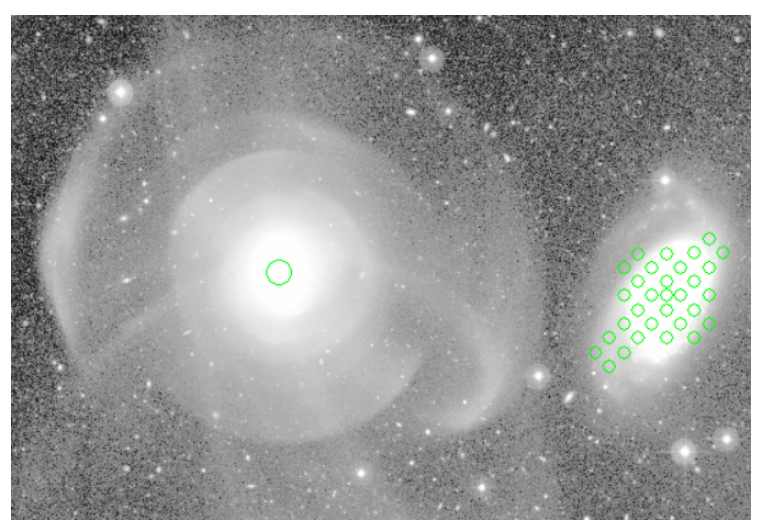

Fig. 2. Image in the $\mathrm{g}$ band from the CFHT Megacam of NGC 474 and companion NGC 470 , enhancing the low surface brightness features, such as the shells (Duc et al. 2015). The CO(1-0) 23" beam is indicated at the center of NGC 474 , and the maps of the $\operatorname{CO}(2-1) 11^{\prime \prime}$ beams cover its companion (see the appendix).

spatial correlation between GCs and stellar fine-structure (shells, tails, radial streams) is remarkable, while GCs are blue, likely to have formed in low-metallicity satellites, and accreted later. In the center of the galaxy, GCs have an intermediate color that is compatible with their formation in situ, when star formation occurred 7-8 Gyr ago. With passive evolution until 13 Gyr, they will become red, as expected in a massive elliptical today (Lim et al. 2017).

\section{3. $\operatorname{Arp} 10$}

This peculiar galaxy reveals loops, ripples, and shells in its outer parts (Charmandaris \& Appleton 1996). The HI VLA map from the latter work shows a neutral gas extension beyond the deepest optical shells. The HI appears in two components: one more relaxed in a rotating disk in the inner parts, although with noncircular motions, and the other component quite similar to a shell system, with no one-to-one correspondence with the optical shells. Bizyaev et al. (2007) showed that the inner parts of the optical disk are dominated by a collisional ring, and the intruder that is responsible for the expanding wave is found $5^{\prime \prime}$ to the southwest from the nucleus of Arp10 (see Fig. 4). Its mass could 


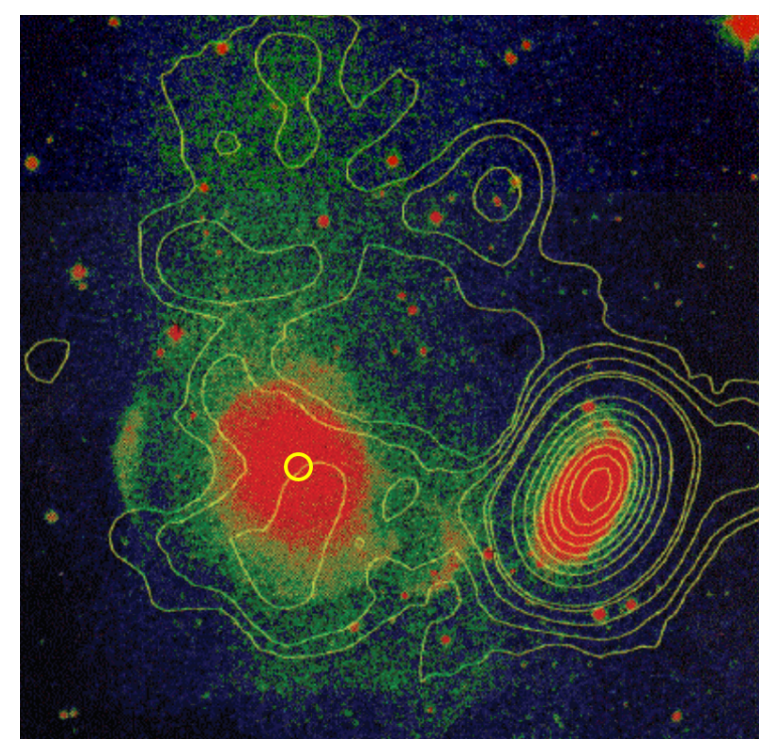

Fig. 3. VLA HI map over Arp 227 (NGC 470 at the right and NGC 474 at the left), from Schiminovich et al. (1997) and van der Hulst (priv. comm.). The $\mathrm{CO}(1-0) 23^{\prime \prime}$ beam is indicated with a yellow circle.

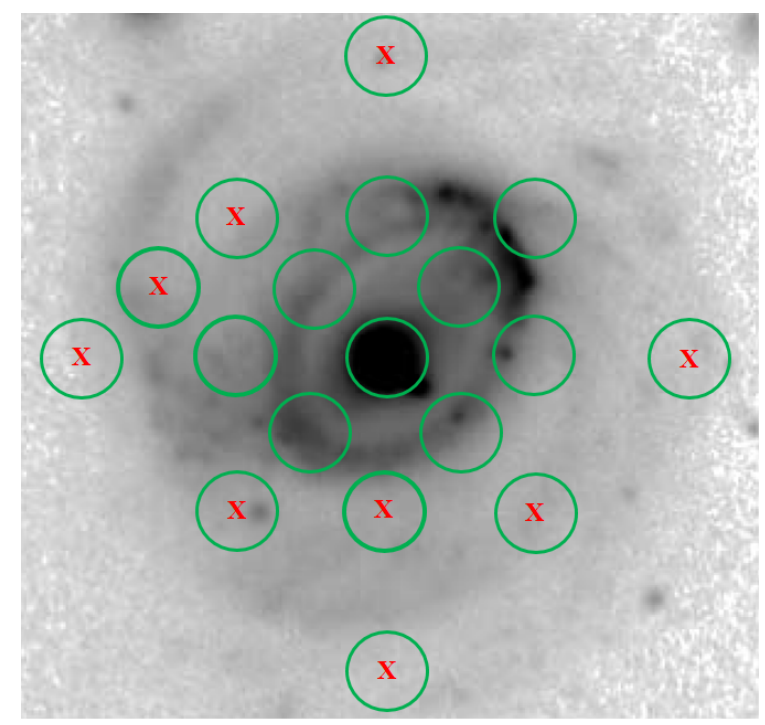

Fig. 4. $C O(2-1)-11^{\prime \prime}$ observed beams in Arp 10, overlaid on the blue optical image, from Bizyaev et al. (2007). The nine pointings selected to be outside the ring are indicated as red crosses (see Fig. 12). They can all be considered to cover part of a shell.

be one-fourth of the mass of Arp10. Star formation has been triggered in the ring by the collision.

\subsection{NGC 3032}

This early-type galaxy is classified as a weakly barred lenticular. Its optical image is very smooth, with some dusty inner disk, however, and a large-scale shell system in which the galaxy is embedded (see Fig. 5 and Duc et al. 2015). This galaxy has certainly acquired its gas recently because the HI map shows counter-rotation with respect to the stars (Oosterloo et al. 2010). While all gas components (ionized, atomic, and molecular) corotate, the main stellar body counter-rotates, but some young stars that have probably formed since the gas accretion event corotate with the gas in the very center (McDermid et al. 2006).

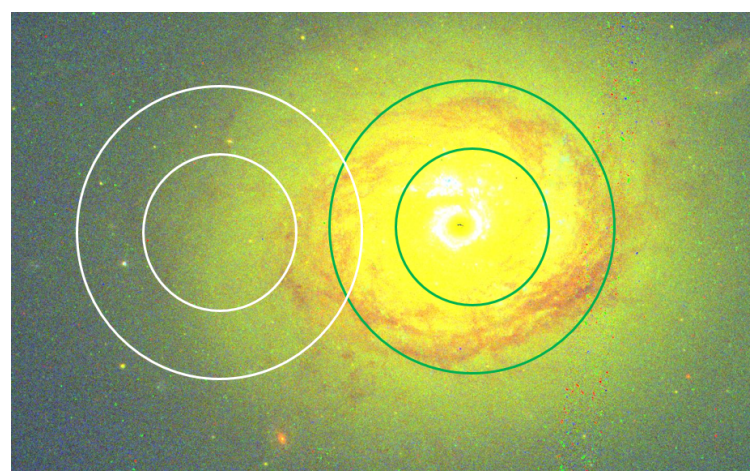

Fig. 5. $\mathrm{CO}(1-0)$ and $\mathrm{CO}(2-1)$ observed beams on NGC 3032, overlaid on an HST composite image. The eastern offset is centered on a shell.

Sage \& Wrobel (1989) detected CO(1-0) emission toward the peculiar lenticular NGC 3032 with the Kitt Peak $12 \mathrm{~m}$ telescope $\left(60^{\prime \prime}\right.$ beam), and derived an $\mathrm{H}_{2}$ mass of $3.6 \times 10^{8} M_{\odot}$, computed with the same distance and CO-to- $\mathrm{H}_{2}$ conversion factor as here. The latter is somewhat higher than that found by Combes et al. (2007), of $2.5 \times 10^{8} M_{\odot}$, but this could be explained because the IRAM- $30 \mathrm{~m}$ beam is $23^{\prime \prime}(2.4 \mathrm{kpc})$ in $\mathrm{CO}(1-0)$ and may miss some outer emission. The Combined Array for Research in Millimeter-Wave Astronomy (CARMA) interferometer mapped the inner disk, but did not cover more than the IRAM-30 $\mathrm{m}$ beam (Alatalo et al. 2013).

\subsection{NGC 3656}

This peculiar galaxy (Arp 55) has been classified as a shell elliptical galaxy, with several shells, and one particularly strong shell $9 \mathrm{kpc}$ south of the center (Balcells et al. 2001), as is shown in Fig. 6. Two kinematical components have been identified, with perpendicular axes (Balcells \& Stanford 1990). The inner (1 kpc) stellar core rotates with a major axis E-W, and the larger stellar and gaseous disk (radius $7 \mathrm{kpc}$ ) rotates with a major axis N-S. The VLA HI map from Balcells et al. (2001) shows two gas components: an edge-on and warped inner disk that extends up to $7 \mathrm{kpc}\left(M(\mathrm{HI})=2 \times 10^{9} M_{\odot}\right)$, aligned north-south along an inner dust lane; and a more extended outer structure, distributed in shells or rings, that is roughly correlated to the optical shells but is more extended. The HI southern shell at $9 \mathrm{kpc}$ coincides with the stellar shell, and has the systemic velocity.

Wiklind et al. (1995) detected CO emission toward the merger system NGC 3656 and derived an $\mathrm{H}_{2}$ mass of $2.2 \times$ $10^{9} M_{\odot}$. A CO map was made with the Berkeley-IllinoisMaryland Association millimeter wave interferometer (BIMA) by Young (2002) and revealed the same N-S alignment as the HI disk. The map was not sensitive enough to detect $\mathrm{CO}$ in the southern shell.

\subsection{NGC 3934}

This peculiar disk galaxy is a candidate polar ring (Whitmore et al. 1990), belonging to a compact group, similar to a Hickson group (Bettoni et al. 2011). It has a strong linear dust lane in the center that irregularly obscures the inner and brighter regions of the galaxy (see Fig. 7). Spurs and shells are visible in the inner parts, but especially in the outskirts of the galaxy. The latter have an average $B-R \sim 1.2$, which is not as blue as typical spiral arms, although they are bluer than the central parts that are reddened by the dust lane. The galaxy has then a rich shell 


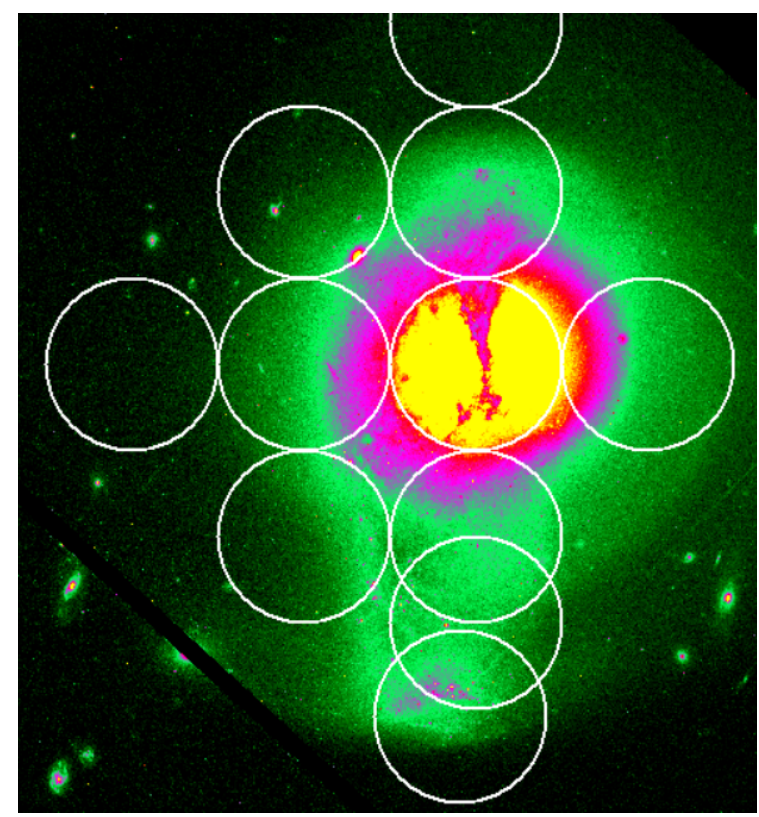

Fig. 6. $\mathrm{CO}(1-0) 23^{\prime \prime}$ beams observed toward NGC 3656, superposed on an F606W HST image. The N-S dust lane is prominent, and the southern shell lies the bottom of the image. All CO beams partially cover some shell, except for the central beam, which includes the dust lane.

system, dispatched all around the center, in apparent random order. This shell morphology can be reproduced in models with oblate galaxies, with shells near the equatorial plane (Dupraz \& Combes 1986). NGC 3934 is rich in gas, it was detected in HI, with a mass of $5 \times 10^{9} M_{\odot}$ (van Driel et al. 2000) and in CO (Galletta et al. 1997) with $M\left(\mathrm{H}_{2}\right)=2.1 \times 10^{9} M_{\odot}$. It has a star formation rate of $\mathrm{SFR}=1.6 M_{\odot} \mathrm{yr}^{-1}$, derived from its far-infrared IRAS flux. Bettoni et al. (2011) proposed a formation in a dissipative merger through hydrodynamical SPH simulations. From this formation mechanism, molecular gas might be expected in the shells.

\subsection{NGC 5018}

Like NGC 7252, NGC 5018 is one of the prototypes of a recent merger, $3 \mathrm{Gyr}$ ago, which is becoming a giant elliptical (gE) (Buson et al. 2004). It is the dominant $\mathrm{gE}$ of a small group. It has been detected by IRAS, with an $\mathrm{SFR}=0.7 M_{\odot} \mathrm{yr}^{-1}$. The galaxy reveals sharp outer shells (Rampazzo et al. 2007) and a central linear dust lane. Kim et al. (1988) mapped the HI, and found $M(\mathrm{HI})=6.1 \times 10^{8} M_{\odot}$. Conversely, the galaxy is not detected in $\mathrm{CO}(1-0)$ with the Atacama Large Millimeter/submillimeter Array (ALMA) (Ueda et al. 2014), with $M\left(\mathrm{H}_{2}\right)<2.3 \times 10^{7} M_{\odot}$, but is detected in the $3 \mathrm{~mm}$ continuum.

\subsection{NGC 7600}

This is a typical shell galaxy with many shells that are aligned and interleaved along the major axis. The main body is a flattened early-type galaxy that is classified as a lenticular. Turnbull et al. (1999) counted 16 shells; the first lies at $40 \mathrm{kpc}$ from the center. Cooper et al. (2011) revealed some even deeper external features, and easily reproduced the system of shells from a simulation of phase wrapping during the radial merger of a small companion. There is only an upper limit for HI in this galaxy (Sansom et al. 2000; Serra et al. 2008). It was selected in our sample, however, because of its high degree of fine stellar structures.

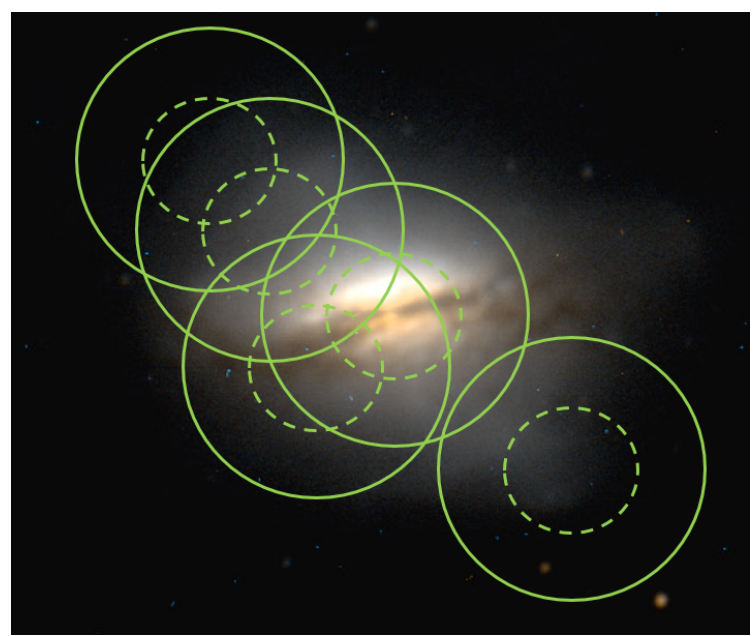

Fig. 7. $\mathrm{CO}(1-0) 23^{\prime \prime}$ and $\mathrm{CO}(2-1) 11^{\prime \prime}$ beams observed toward NGC 3934, overlaid on a composite optical image from the SDSS. All offset beams cover some shells, which are expected to show plateau emission before a sharp edge.

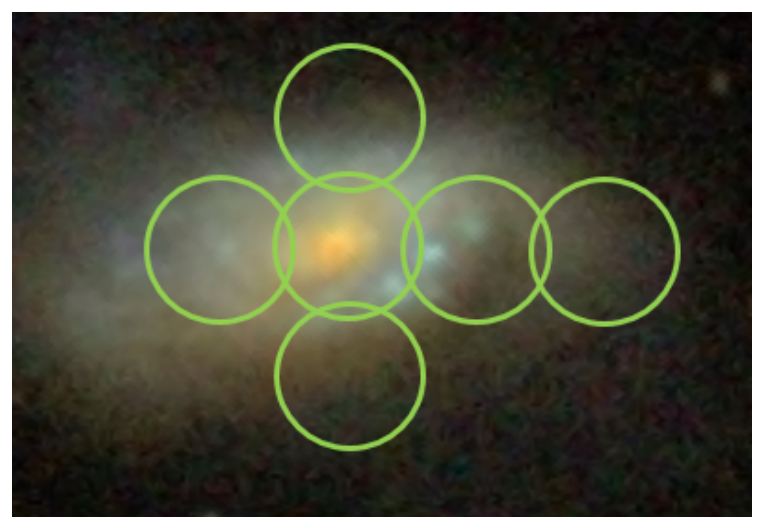

Fig. 8. $\mathrm{CO}(2-1) 11^{\prime \prime}$ beams observed toward Arp 295N, overlaid on a composite SDSS image. The western offsets cover shells.

\section{9. $\operatorname{Arp} 295 \mathrm{~N}$}

The system Arp 295 is a pair of galaxies in an early merger stage. The northern member reveals large plumes, shells, and loops of $\mathrm{H} \alpha$ emission up to $9 \mathrm{kpc}$ north and south of the minor axis (see Fig. 8). The northern galaxy is undergoing a retrograde encounter with its southern companion. The HI was mapped with the VLA by Hibbard \& van Gorkom (1996), who found an HI mass of $1.7 \times 10^{10} M_{\odot}$ and an SFR $=17 M_{\odot} \mathrm{yr}^{-1}$. They also reported our IRAM-30 m observations of $\mathrm{CO}$ emission, which were not published, with a molecular mass of $M\left(\mathrm{H}_{2}\right)=8.5 \times$ $10^{9} M_{\odot}$. The SFR of the ensemble has been estimated to be 21.9 $M_{\odot} \mathrm{yr}^{-1}$ from Pa $\alpha$ by Tateuchi et al. (2015).

\section{Results}

In each shell galaxy, the center was observed first to determine whether it contained molecular gas, and if present, the shell regions were also observed. Gas in shells is expected to come from the infall of a gas-rich satellite (Charmandaris et al. 2000; Combes \& Charmandaris 2000). The gas in the satellite is first liberated and may oscillate in the potential of the primary galaxy, just before the satellite stars form the shells. Some gas is expected to fall in the center through dissipation. Gas in the 
Table 2. CO results.

\begin{tabular}{|c|c|c|c|c|c|c|c|c|c|}
\hline \multirow[b]{2}{*}{ Galaxy } & \multirow[b]{2}{*}{$\begin{array}{l}\text { Offsets } \\
\left({ }^{\prime \prime},{ }^{\prime \prime}\right)\end{array}$} & \multicolumn{3}{|c|}{$\mathrm{CO}(1-0)$} & \multicolumn{3}{|c|}{$\mathrm{CO}(2-1)$} & \multirow[b]{2}{*}{$\begin{array}{l}M\left(\mathrm{H}_{2}\right) \\
10^{8} M_{\odot}\end{array}$} & \multirow[b]{2}{*}{$\begin{array}{c}\Sigma\left(\mathrm{H}_{2}\right) \\
M_{\odot} \mathrm{pc}^{-2}\end{array}$} \\
\hline & & $\begin{array}{c}\text { Int-flux } \\
\mathrm{Jy} \mathrm{km} \mathrm{s}^{-1}\end{array}$ & $\begin{array}{c}V \\
\mathrm{~km} \mathrm{~s}^{-1} \\
\end{array}$ & $\begin{array}{c}\Delta V \\
\mathrm{~km} \mathrm{~s}^{-1}\end{array}$ & $\begin{array}{c}\text { Int-flux } \\
\mathrm{Jy} \mathrm{km} \mathrm{s}^{-1}\end{array}$ & $\begin{array}{c}V \\
\mathrm{~km} \mathrm{~s}^{-1}\end{array}$ & $\begin{array}{c}\Delta V \\
\mathrm{~km} \mathrm{~s}^{-1}\end{array}$ & & \\
\hline \multirow[t]{3}{*}{ ARP 230} & $(0,0)$ & $9.2 \pm 0.9$ & $-54 \pm 6$ & $125 \pm 15$ & $14.7 \pm 1$ & $-42 \pm 5$ & $111 \pm 11$ & $0.57 \pm 0.06$ & 11 \\
\hline & $(4.4,17)$ & $3.7 \pm 0.7$ & $5 \pm 17$ & $190 \pm 40$ & $<3$ & - & - & $0.23 \pm 0.04$ & 4 \\
\hline & $(-14,-21)$ & $<1.6$ & - & - & $<1.7$ & - & - & $<0.1$ & $<2$ \\
\hline NGC 474 & $(0,0)$ & $<1.4$ & - & - & $<3.1$ & - & - & $<0.15$ & $<1.6$ \\
\hline \multirow[t]{2}{*}{ ARP 10} & $(0,0)$ & $29.7 \pm 1$ & $10 \pm 4$ & $208 \pm 7$ & $38.8 \pm 2.7$ & $25 \pm 8$ & $224 \pm 16$ & $44.6 \pm 1.5$ & 35 \\
\hline & See & $18 \mathrm{pt}$ & Map & & & & & & \\
\hline \multirow[t]{2}{*}{ NGC 3032} & $(0,0)$ & $71 \pm 1.3$ & $57 \pm 1$ & $129 \pm 2$ & $86.5 \pm 2$ & $47 \pm 1$ & $108 \pm 3$ & $3.6 \pm 0.06$ & 90 \\
\hline & $(20,0)$ & $8 \pm 0.8$ & $80 \pm 3$ & $53 \pm 6$ & $<5$ & - & - & $0.4 \pm 0.04$ & 10 \\
\hline \multirow[t]{9}{*}{ NGC 3656} & $(0,0)$ & $119.6 \pm 2.2$ & $-34 \pm 4$ & $433 \pm 8$ & $200 \pm 4.5$ & $-31 \pm 7$ & $407 \pm 23$ & $22.9 \pm 0.41$ & 142 \\
\hline & $(22,22)$ & $9.2 \pm 1.3$ & $-192 \pm 8$ & $112 \pm 17$ & $29.8 \pm 4.0$ & $6 \pm 10$ & $322 \pm 45$ & $1.7 \pm 0.25$ & 11 \\
\hline & $(0,22)$ & $9.4 \pm 1.6$ & $-184 \pm 12$ & $153 \pm 33$ & $<8$ & - & - & $1.8 \pm 0.3$ & 11 \\
\hline & $(22,0)$ & $47.6 \pm 2.0$ & $-20 \pm 9$ & $433 \pm 18$ & $2.7 \pm 0.8$ & $-78 \pm 8$ & $51 \pm 19$ & $9.1 \pm 0.4$ & 57 \\
\hline & $(22,-22)$ & $8.6 \pm 1.7$ & $107 \pm 14$ & $143 \pm 32$ & $5.7 \pm 1.0$ & $106 \pm 6$ & $73 \pm 14$ & $1.64 \pm 0.3$ & 10 \\
\hline & $(0,-22)$ & $18.6 \pm 1.5$ & $130 \pm 9$ & $232 \pm 23$ & $<7$ & - & - & $3.55 \pm 0.3$ & 22 \\
\hline & $(0,-33)$ & $3.6 \pm 1.0$ & $126 \pm 18$ & $135 \pm 46$ & $4.8 \pm 1.0$ & $91 \pm 16$ & $149 \pm 30$ & $0.69 \pm 0.2$ & 4.3 \\
\hline & $(2,-45)$ & $1.5 \pm 0.3$ & $179 \pm 15$ & $139 \pm 29$ & $2.5 \pm 0.8$ & $105 \pm 34$ & $187 \pm 67$ & $0.29 \pm 0.06$ & 1.8 \\
\hline & See & $11 \mathrm{pt}$ & Map & & & & & & \\
\hline \multirow[t]{5}{*}{ NGC 3934} & $(0,0)$ & $42.8 \pm 0.8$ & $22 \pm 4$ & $384 \pm 7$ & $78.9 \pm 1.7$ & $30 \pm 4$ & $396 \pm 9$ & $11 \pm 0.2$ & 51 \\
\hline & $(7,-5)$ & $21.4 \pm 0.8$ & $-17 \pm 7$ & $350 \pm 13$ & $18.7 \pm 3$ & $-78 \pm 19$ & $210 \pm 67$ & $5.5 \pm 0.2$ & 25 \\
\hline & $(16,14)$ & $3.7 \pm 0.4$ & $-128 \pm 7$ & $115 \pm 17$ & $1.2 \pm 0.4$ & $-119 \pm 17$ & $86 \pm 27$ & $0.9 \pm 0.1$ & 4 \\
\hline & $(-16,-14)$ & $6.6 \pm 1$ & $134 \pm 18$ & $255 \pm 77$ & $3 \pm 0.5$ & $184 \pm 7$ & $90 \pm 19$ & $1.7 \pm 0.2$ & 8 \\
\hline & $(11,12)$ & $<10$ & - & - & $<90$ & - & - & $<2.5$ & $<11$ \\
\hline \multirow[t]{3}{*}{ NGC 5018} & $(0,0)$ & $<1.8$ & - & - & $<4$ & - & - & $<0.27$ & $<2$ \\
\hline & $(44,-22)$ & $<3$ & - & - & $<4$ & - & - & $<0.45$ & $<3.6$ \\
\hline & $(-22,22)$ & $<3$ & - & - & $<3$ & - & - & $<0.45$ & $<3.6$ \\
\hline \multirow[t]{2}{*}{ NGC 7600} & $(0,0)$ & $<3$ & - & - & $<4.8$ & - & - & $<0.5$ & $<3.6$ \\
\hline & $(143,32)$ & $<2$ & - & - & $<5$ & - & - & $<0.3$ & $<2$ \\
\hline \multirow[t]{4}{*}{ ARP 295N } & $(0,0)$ & $58 \pm 4$ & $-54 \pm 11$ & $333 \pm 24$ & $38 \pm 5$ & $-42 \pm 20$ & $268 \pm 40$ & $53 \pm 4$ & 69 \\
\hline & $(-10,0)$ & $45 \pm 3.8$ & $16 \pm 14$ & $340 \pm 33$ & $25.8 \pm 5.6$ & $85 \pm 14$ & $136 \pm 35$ & $41 \pm 3.4$ & 53 \\
\hline & $(-20,0)$ & $7.6 \pm 2.2$ & $110 \pm 13$ & $93 \pm 35$ & $<2$ & - & - & $6.9 \pm 2$ & 9 \\
\hline & See & $6 \mathrm{pt}$ & Map & & & & & & \\
\hline
\end{tabular}

Notes. Integrated flux, velocity, and FWHM obtained through Gaussian fits. Velocities are counted from the central redshifts indicated in Table 1. The $\mathrm{H}_{2}$ masses are estimated within the $\mathrm{CO}(1-0)$ beam of $23^{\prime \prime}$, with the standard conversion factor of the Milky Way $\alpha=4.36 M_{\odot}\left(\mathrm{K} \mathrm{km} \mathrm{s}^{-1} \mathrm{pc}^{2}\right)^{-1}$. Upper limits are given at $3 \sigma$, assuming an FWHM of $300 \mathrm{~km} \mathrm{~s}^{-1}$.

center is therefore a necessary condition for finding gas in shells, but is not sufficient because the primary galaxy could also have had some gas before the collision. Out of the nine shell galaxies of our sample, three were not detected in their center (NGC 474, NGC 5018, and NGC 7600) and we did not search for gas in their shells. The upper limits are displayed in Table 2. Three of the six remaining shell galaxies were mapped extensively, and we show spectra maps in $\mathrm{CO}(1-0)$ and $\mathrm{CO}(2-1)$ for Arp 10 (Figs. 10 and 11), NGC 3656 (Fig. 17), and Arp 295N in Figs. 19 and 20. In Table 2 we characterize only the central beam and the offset beams that cover a shell and are detected in $\mathrm{CO}(1-0)$. For the three other galaxies (Arp 230, NGC 3032, and NGC 3934), only a few spectra are shown in Figs. 9, 14, and 18. All these points are detailed in Table 2.

\subsection{Molecular gas content}

To quantify the amount of molecular gas found in each pointing, we relied on the empirically established proportionality between the $\mathrm{CO}(1-0)$ luminosity and $\mathrm{H}_{2}$ mass for a large number of Milky Way-like galaxies (e.g., Bolatto et al. 2013). We converted the integrated intensities in each beam (in $T_{\mathrm{mb}}$ ) into integrated fluxes $S(\mathrm{CO}) \mathrm{d} V$, with the conversion of $5 \mathrm{Jy}$ per Kelvin, as appropriate for the IRAM-30 m telescope. From the integrated flux $S(\mathrm{CO}) \mathrm{d} V\left(\mathrm{Jy} \mathrm{km} \mathrm{s}^{-1}\right)$ found within each region, the derived molecular mass was obtained through the formula

$L_{\mathrm{CO}}^{\prime}\left(\mathrm{K} \mathrm{km} \mathrm{s}^{-1} \mathrm{pc}^{-2}\right)=3.25 \times 10^{7} \frac{S(\mathrm{CO}) \mathrm{d} V}{(1+z)}\left(\frac{D_{\mathrm{L}}}{v_{\text {rest }}}\right)^{2}$,

where $v_{\text {rest }}=115.271 \mathrm{GHz}$, and $D_{\mathrm{L}}$ is the luminosity distance in megaparsec (e.g., Solomon \& Vanden Bout 2005). The molecular mass, including helium, was then derived from

$M\left(\mathrm{H}_{2}\right)=4.36 L_{\mathrm{CO}}^{\prime}$

assuming the standard CO-to- $\mathrm{H}_{2}$ conversion factor of $X_{\mathrm{CO}}=2 \times$ $10^{20} \mathrm{~cm}^{-2}\left(\mathrm{~K} \mathrm{~km} \mathrm{~s}^{-1}\right)^{-1}$, as applicable to Milky Way-like galaxies. Although shell galaxies are interacting, they are mildly perturbed and are certainly not starbursts, or ultra-luminous infrared galaxies (ULIRG), where a lower conversion factor might apply.

Molecular masses, corresponding to each $\mathrm{CO}(1-0)$ pointing are displayed in Table 2. In most cases, we did not observe the whole galaxy disks, and an estimation of the total molecular masses would need some extrapolation. 


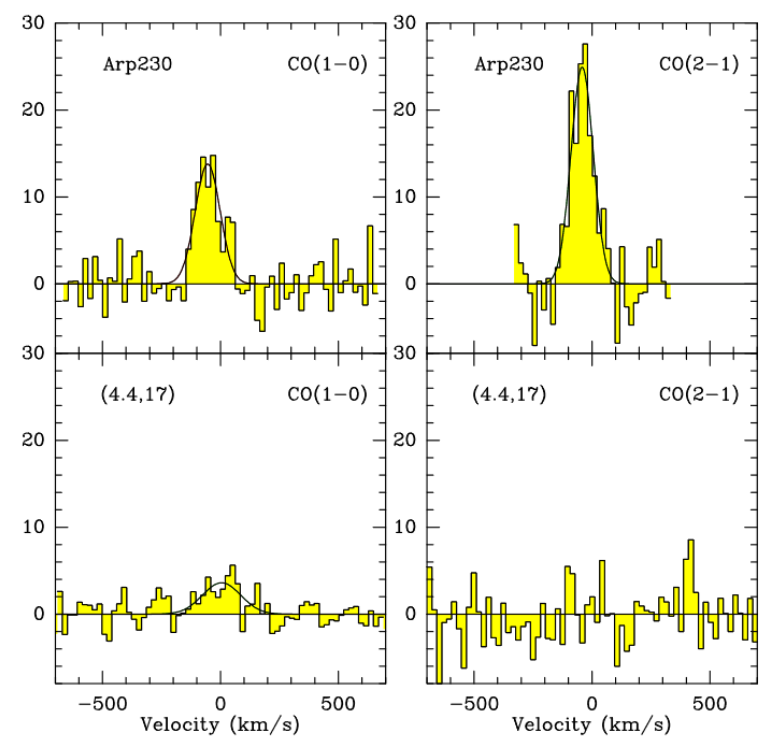

Fig. 9. CO spectra in Arp 230, central position and offset (in arcsec). The vertical axis is the main-beam temperature in $\mathrm{mK}$.

The last column of Table 2 reports the average molecular surface density within the $\mathrm{CO}(1-0)$ beam of $23^{\prime \prime}$ for each galaxy. This shows that galaxies of later type (e.g., NGC 3656 or NGC 3934) tend to have a richer content in molecular gas, which might be due to their own gas before the accretion event. We verified that the total molecular content is correlated to the farinfrared (FIR) luminosity (see Table 1), and that galaxies of our sample are compatible with the global Kennicutt-Schmidt (KS) diagram, as shown for local early-type galaxies by Combes et al. (2007). However, we have not enough sensitivity in $\mathrm{H} \alpha$ or farultraviolet (FUV) or near-ultraviolet (NUV) (GALEX) images to be able to determine the star formation surface density on shells, and compare this to the molecular surface densities to study the KS diagram of shells.

\subsection{CO spectra}

For most of the shell galaxies with detected in $\mathrm{CO}$ emission at least in their center, we tried to cover the outer stellar shells through simultaneous pointing in the $\mathrm{CO}(1-0)$ and $\mathrm{CO}(2-1)$ lines. Shells are not only reduced to their sharp edges, but also extend inside these arcs in a nearly flat plateau emission, as we show in Fig. 2 for example. With the large CO beams, we can thus hope to collect emission from these regions as well. The best resolution we have, 11 arcsec, typically corresponds to 1$5 \mathrm{kpc}$ in the galaxies, and is just sufficient to separate the shell regions from the center, while the $23^{\prime \prime}$ beam is not. Frequently, the $\mathrm{CO}(1-0)$ emission is detected in the shell offset position, but the $\mathrm{CO}(2-1)$ is not, which can be interpreted as the absence of detection in the shell itself. The $\mathrm{CO}(1-0)$ emission is then only due to some central emission seen at the border of the beam.

This is clearly the case for Arp 230, as is shown in Fig. 9. $\mathrm{CO}$ emission towards the center is highly concentrated, which results in the peak main-beam temperature being nearly twice in $\mathrm{CO}(2-1)$ with respect to $\mathrm{CO}(1-0)$. The offset beam, at $17.5^{\prime \prime}=$ $2 \mathrm{kpc}$ from the center, is detected in $\mathrm{CO}(1-0)$ but is consistent with emission from the central disk. The offset is not detected in $\mathrm{CO}(2-1)$, whose beams does not overlap with the disk. Ueda et al. (2014) have mapped the $\mathrm{CO}(1-0)$ emission with ALMA with 4 arcsec resolution and showed that it is confined in a thin rotating edge-on disk, $5 \mathrm{kpc}$ in diameter, corresponding to the

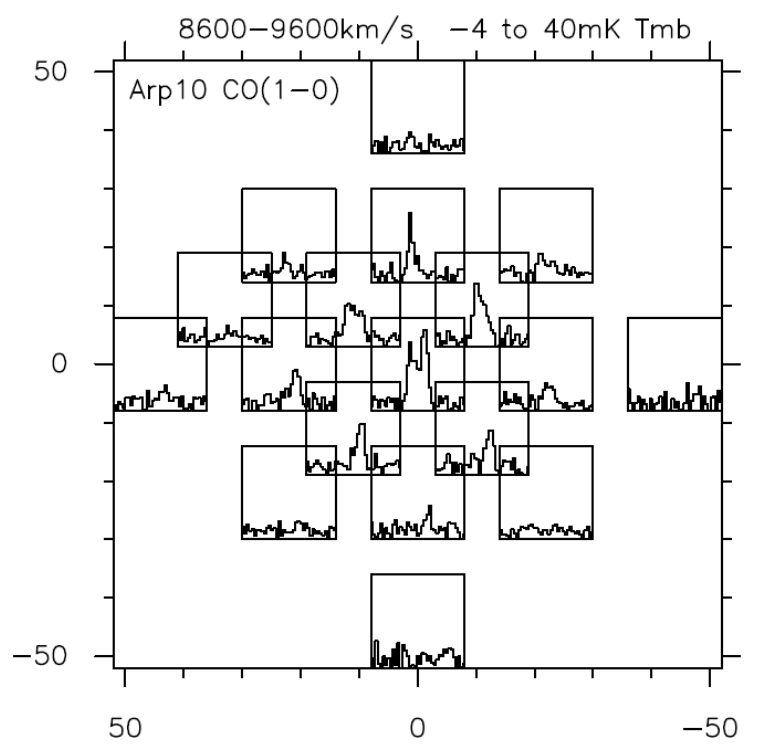

Fig. 10. CO(1-0) map of spectra in Arp 10. The RA and Dec offsets are in arcsec. The scales of all spectral boxes in velocity and main-beam temperature are indicated at the top.

dust lane that is prominent in Fig. 1. Because no emission at all is detected in the farthest shell, at $3 \mathrm{kpc}$ from the center, we conclude that there no gas can be detected in the shells.

The case of Arp 10 is different because the collision at the origin of the shells also produced a collisional ring in the center, that is, a wave propagating radially outward from the center as a result of the excitation of a head-on collision with a small impact parameter (e.g., Appleton \& Struck-Marcell 1996). In the $\mathrm{CO}(2-1)$ map of spectra of Fig. 11, the emission is clearly extended, not only in the ring, but also in the shells. The comparison between the two lines (Figs. 10 and 11) suggests that the gas has indeed been detected as far as $\sim 26 \mathrm{kpc}$ from the center in ripples and shells caused by the interaction. To better quantify the amount of gas involved, we have stacked all the spectra that lie clearly outside the ring: the nine offsets considered are indicated by red crosses in Fig. 4. These nine offsets correspond to the shells. The resulting stacked spectra of the $\mathrm{CO}(1-0)$ and $\mathrm{CO}(2-$ 1) lines are plotted in Fig. 12. All these positions outside the ring were stacked, and their velocity was recentered around the systemic velocity of $9100 \mathrm{~km} \mathrm{~s}^{-1}$. The resulting $\mathrm{CO}(2-1)$ spectrum is the most significant because of the smaller beam, which excludes overlap with the center. The strength of the $\mathrm{CO}(2-1)$ is similar to and even stronger than that of $\mathrm{CO}(1-0)$, which signifies molecular gas outside the ring in the shells. From this stacked spectrum, we derived a molecular content of $M\left(\mathrm{H}_{2}\right)=3.4 \times 10^{9} M_{\odot}$ in the shells, assuming the conversion factor adopted in Sect. 4.1. An overview of the $\mathrm{CO}(1-0)$ and $\mathrm{CO}(2-1)$ surface densities and velocity fields is displayed in Fig. 13. The velocity gradient is comparable to that of the ionized gas, and is limited because the inclination is almost face-on $\left(i=22^{\circ}\right)$ (Bizyaev et al. 2007).

The case of NGC 3032 is quite similar to the map shown in Arp 230. Figure 14 is typical of a quite concentrated CO emission, with the main-beam temperature of the $\mathrm{CO}(2-1)$ line peaking higher than that of the $\mathrm{CO}(1-0)$. The offset shell region does not overlap the center with the 11 arcsec beam, and no $\mathrm{CO}(2-1)$ is detected there, while the $\mathrm{CO}(1-0)$ line shows the redshifted part of the emission, compatible with the inner rotating disk, which is expected to coincide with the dust lanes (see Fig. 5). We conclude that the shell region is not detected in the molecular gas. 


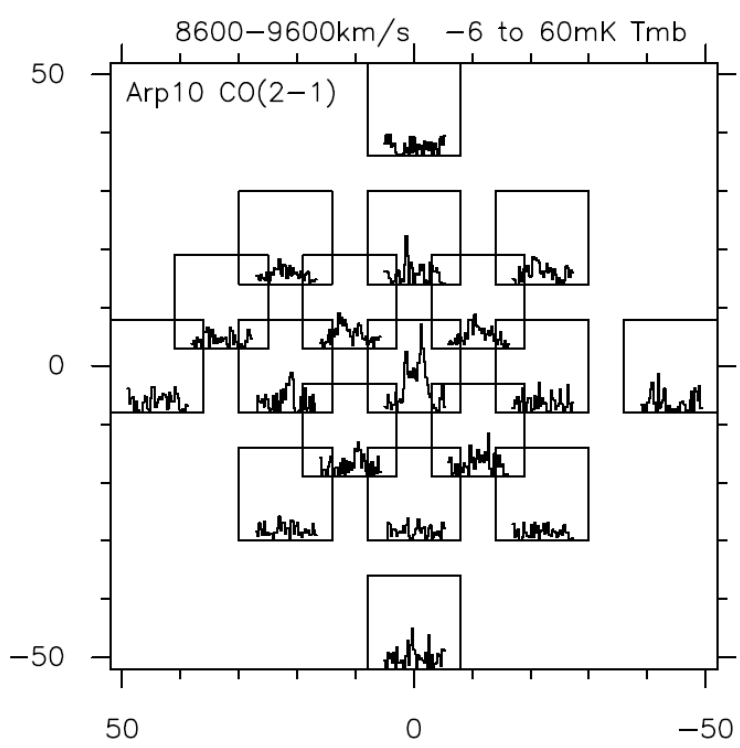

Fig. 11. $\mathrm{CO}(2-1)$ map of spectra in Arp 10.

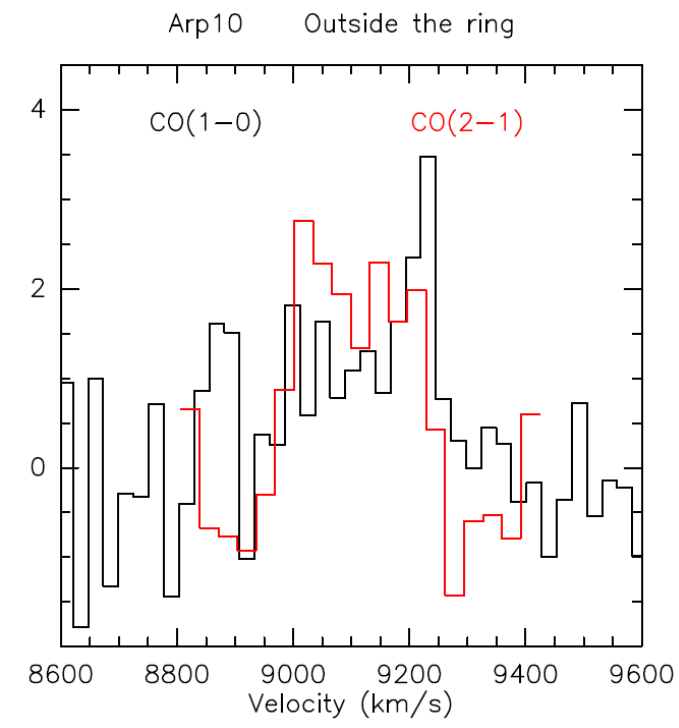

Fig. 12. Stacked CO spectra in Arp 10, considering only the positions outside the ring that cover the shells (see Fig. 4). The vertical axis is the main-beam temperature in $\mathrm{mK}$.

The shell galaxy NGC 3656 appears to be one of the best cases of an elliptical galaxy that has accreted a companion and has formed shells through phase wrapping (Balcells et al. 2001). The accreted companion must have been rich in gas because it left a N-S dust lane that is terminated by the southern shell at $9 \mathrm{kpc}$. This southern shell is clearly detected in molecular gas, as seen through the comparison of the $\mathrm{CO}(2-1)$ and $\mathrm{CO}(1-0)$ spectra in Fig. 15 and the corresponding map (Fig. 17). There are also shells in the outer north, east, and west observed beams, which show $\mathrm{CO}(1-0)$ emission, but no clear $\mathrm{CO}(2-1)$ (Fig. 16 and Table 2). $\mathrm{CO}(1-0)$ and $\mathrm{CO}(2-1)$ are simultaneously clearly detected only in the $(22,22),(22,-22)$, and $(0,-33)$ positions. The velocity of the $\mathrm{CO}$ gas in the shells spans a range around the systemic velocity, as does the velocity of stars in the southern shell (Balcells et al. 2001). In the extremity of the southern shell, the velocity profile is broad: it shows a double-horn shape from 2700 to $3100 \mathrm{~km} \mathrm{~s}^{-1}$ at $(0,-33)$ and a single component from 2900 to $3100 \mathrm{~km} \mathrm{~s}^{-1}$ at $(2,-45)$ in $\mathrm{CO}(1-0)$. In other places, it also shows a two-horn shape, which is usually only seen in

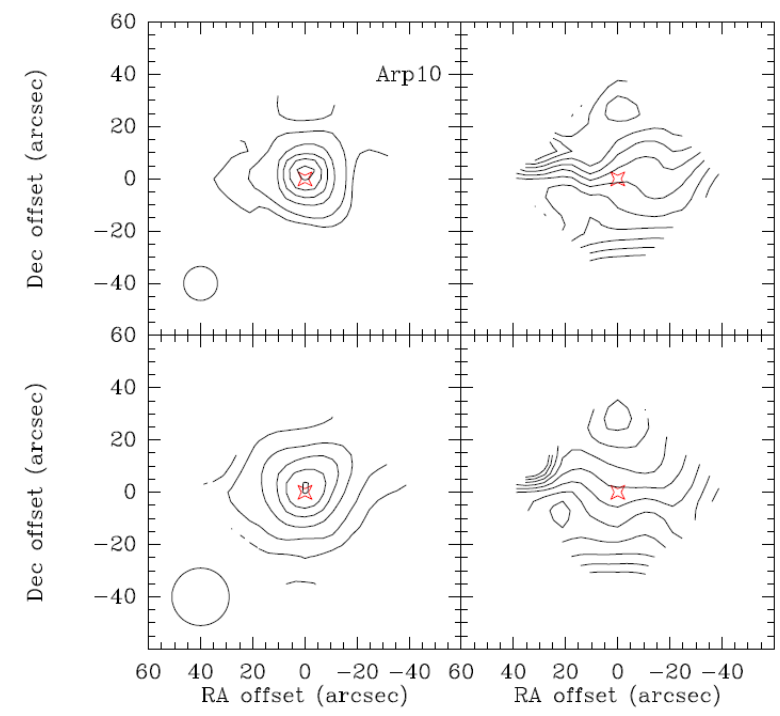

Fig. 13. Isocontours of the integrated intensities in $\operatorname{Arp} 10$ for $\mathrm{CO}(2-1)$ (upper panels) and $\mathrm{CO}(1-0)$ (lower panels), with linear contours spaced by 1.52 and $1.15 \mathrm{~K} \mathrm{~km} \mathrm{~s}^{-1}$, respectively, and maximum contours at 7.6 and $5.7 \mathrm{~K} \mathrm{~km} \mathrm{~s}^{-1}$, respectively. The right panels display the corresponding velocity fields. The isovels are spaced by $30 \mathrm{~km} \mathrm{~s}^{-1}$, they start at $8990 \mathrm{~km} \mathrm{~s}^{-1}$ in the north and extend to $9290 \mathrm{~km} \mathrm{~s}^{-1}$ in the south. The beam sizes are indicated in the bottom left corner.
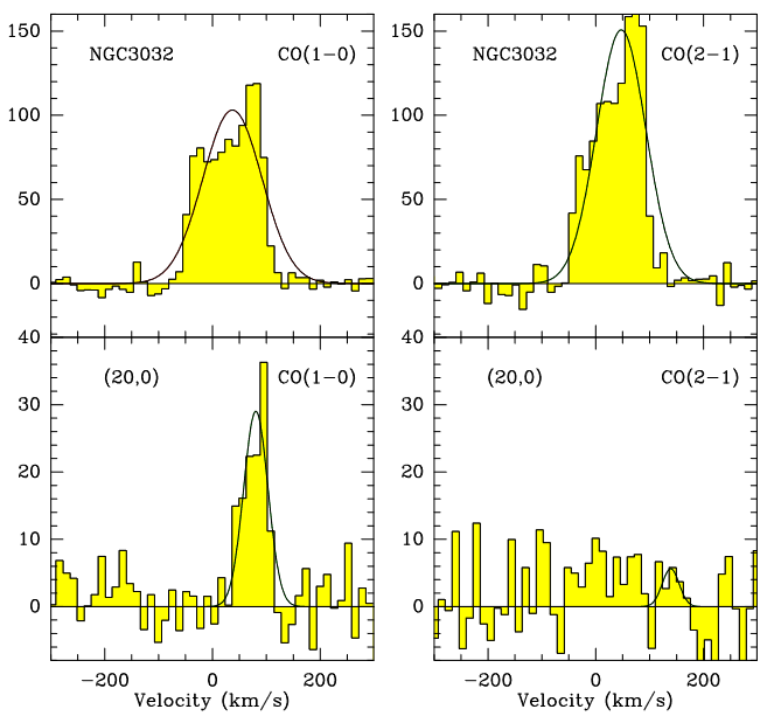

Fig. 14. CO spectra in NGC 3032, central position and offset (in arcsec). The vertical axis is the main-beam temperature in $\mathrm{mK}$.

the central beam for rotating disks, but is not compatible with a rotating disk in the outer parts. This means that two components with different angular momentum exist. The HI component also shows a trend of counter-rotation, as claimed by Balcells et al. (2001). The inner disk does rotate, however: it is redshifted in the south and blueshifted in the north, as is the HI disk observed by Balcells et al. (2001). The fact that the $\mathrm{CO}$ emission observed toward the southern shell has a velocity different from what is expected from the disk rotation confirms its origin in a phasewrapped shell.

Averaging over the three observed beams that cover the southern shell, we obtain a surface density of $\Sigma=9 M_{\odot} \mathrm{pc}^{-2}$ and we derive a molecular content of $M\left(\mathrm{H}_{2}\right)=3.8 \times 10^{8} M_{\odot}$ in the $9 \mathrm{kpc}-$ shell assuming the conversion factor adopted in Sect. 4.1. 


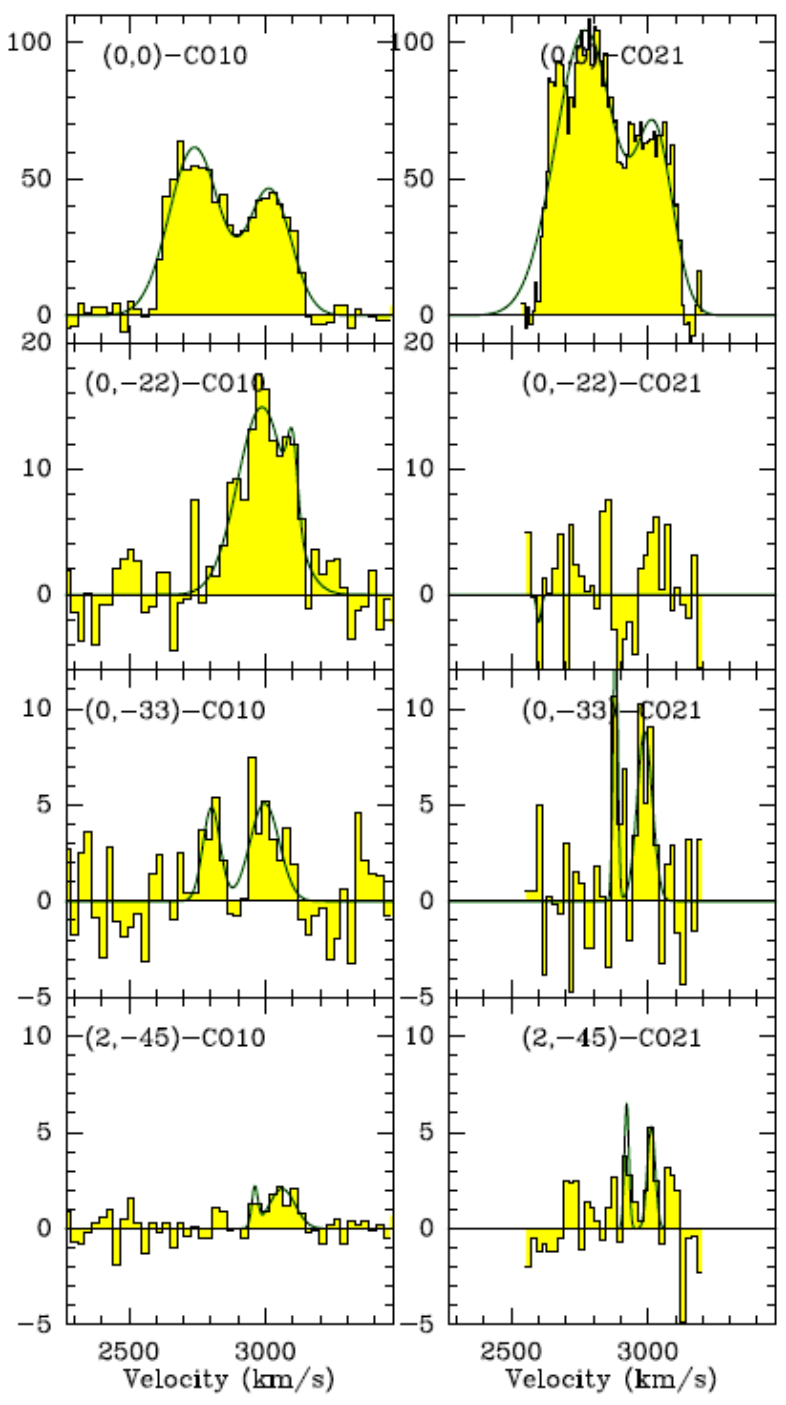

Fig. 15. CO spectra in NGC 3656 for the center and the offsets (in arc$\mathrm{sec})$, covering the southern shell. The vertical axis is the main-beam temperature in $\mathrm{mK}$.

NGC 3934 is a late-type galaxy with randomly oriented shells around a nearly edge-on disk that is traced by a linear dust lane. It has been confused with a polar ring galaxy, but this is not the correct classification (Bettoni et al. 2011). The beams clearly point to the shells, and do not overlap with the disk (see Fig. 7). With symmetrical offsets of $21^{\prime \prime}=5 \mathrm{kpc}$ from the center, they are clearly detected in both $\mathrm{CO}(1-0)$ and $\mathrm{CO}(2-1)$, as shown in Fig. 18. Therefore molecular gas is detected in the shells. There is no shift in average velocity for the gas in the shells with respect to the disk. However, the line profiles in the shells show a twohorn shape, indicating two opposite signs of the relative angular momentum of the shell material relative to the primary galaxy. This two-horn shape is frequently observed toward the center of galaxies and is characteristic of a disk in rotation. The reason is that the observing beam covers both the receding (red) and approaching (blue) sides. In the outer parts, only one velocity component is expected for a rotating disk. A two-horn shape in the outer profiles supports the gas-shell scenario. That the ratios of $\mathrm{CO}(2-1)$ to $\mathrm{CO}(1-0)$ vary from one position to the other is due to the different beams and helps to distinguish the possible contamination in the larger $\mathrm{CO}(1-0)$ beam.

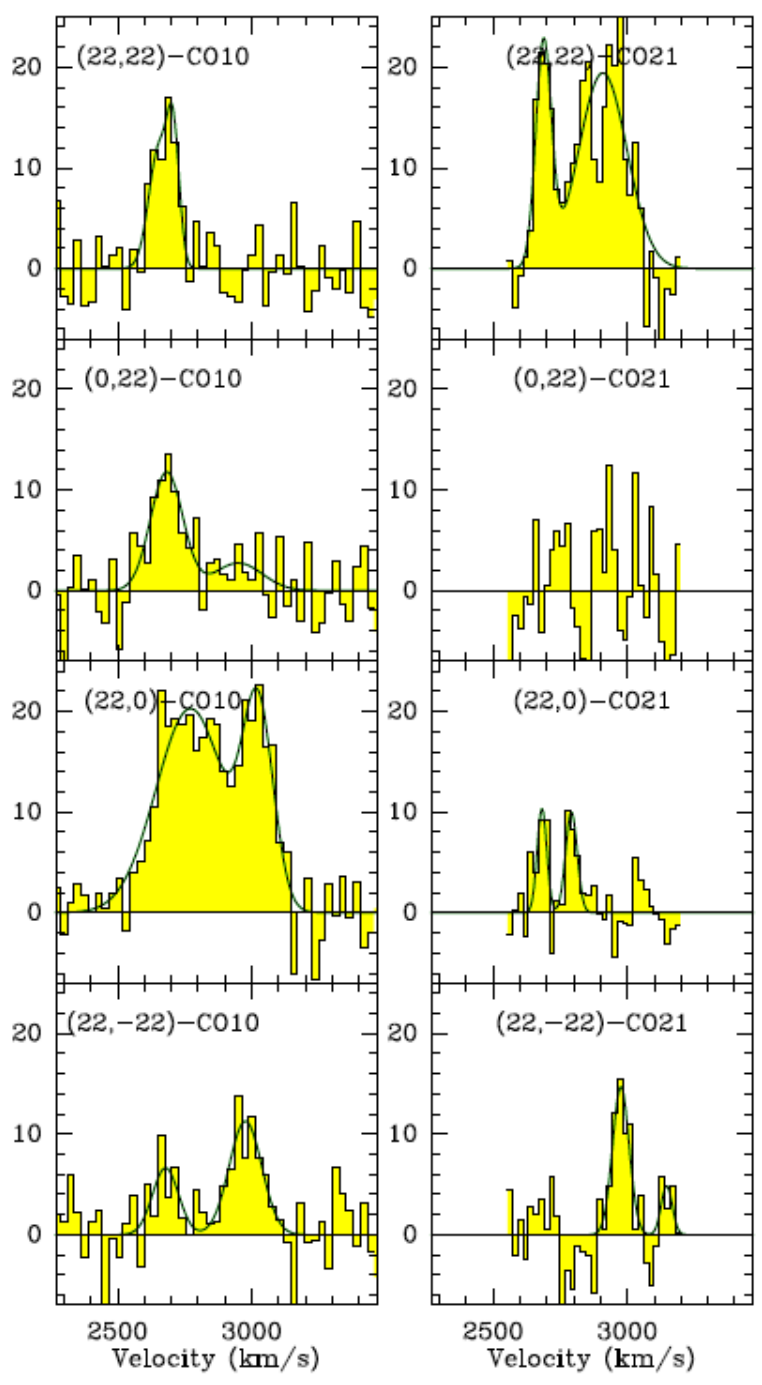

Fig. 16. Same as Fig. 15 for the offsets of NGC 3656 that cover a shell (other than the southern one), and detected in $\mathrm{CO}(1-0)$.

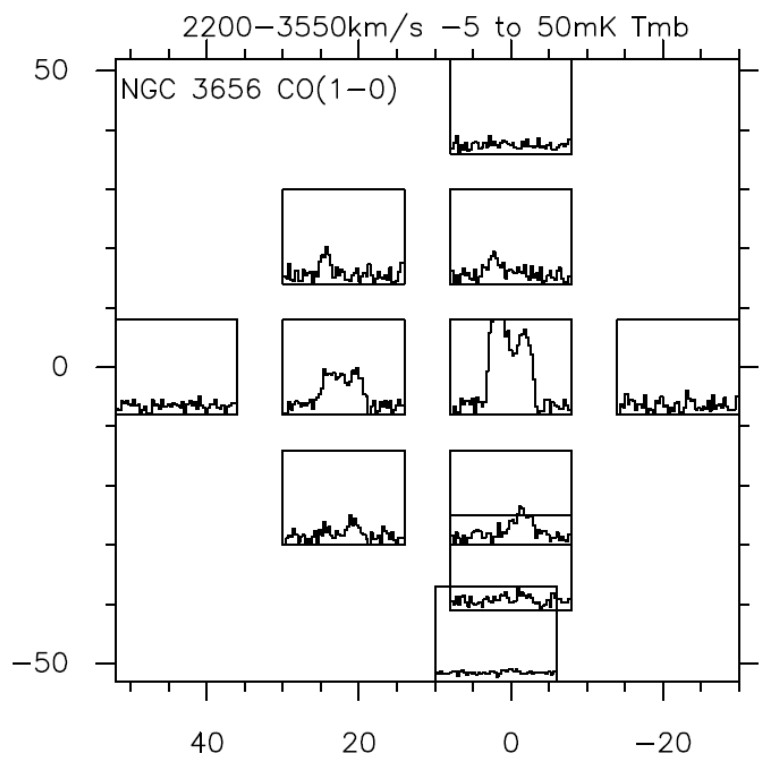

Fig. 17. $\mathrm{CO}(1-0)$ map of spectra in NGC 3656 . The $\mathrm{CO}$ emission is most visible in the southern shell, but is visible in the other three directions. 


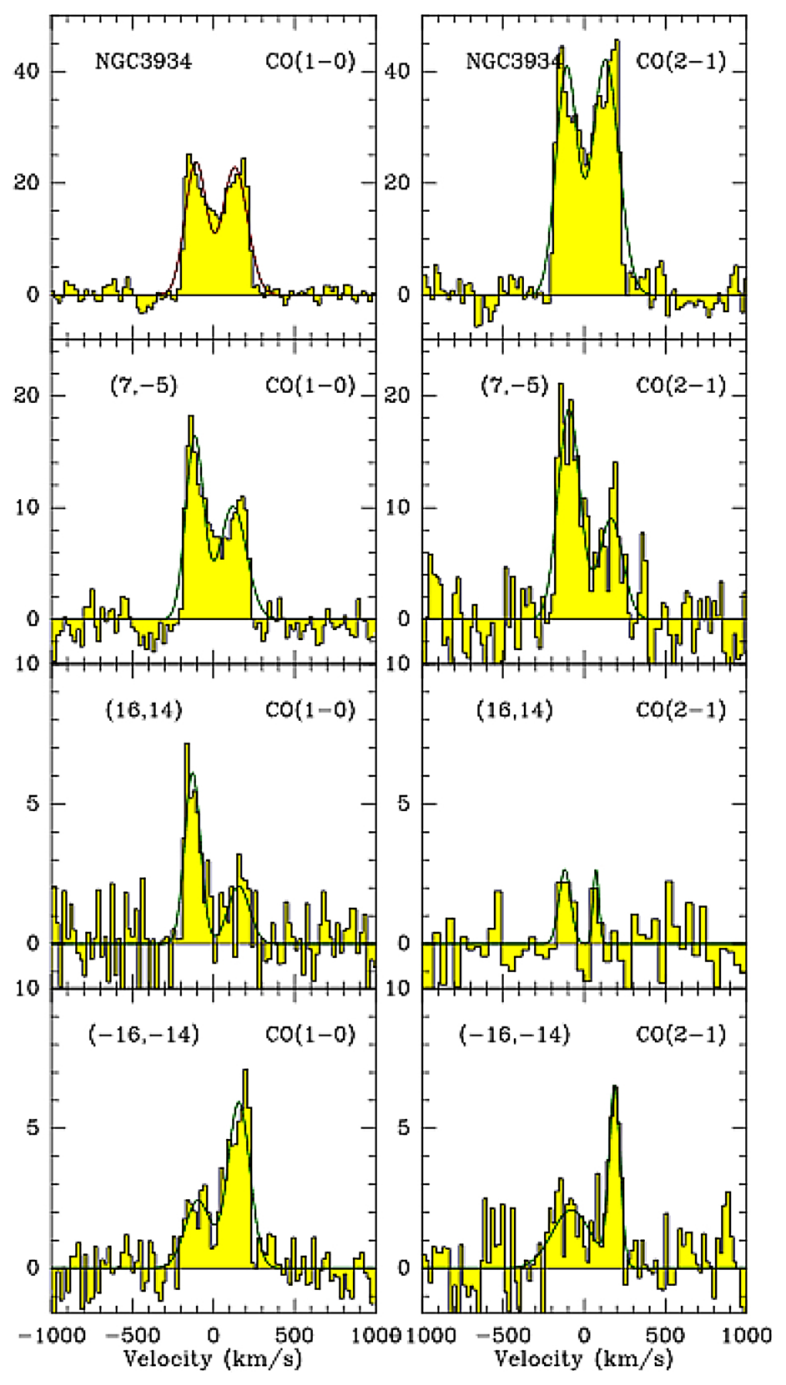

Fig. 18. $\mathrm{CO}(1-0)($ left $)$ and $\mathrm{CO}(2-1)($ right) spectra in NGC 3934, central position and offsets (in arcsec). The vertical axis is the main-beam temperature in $\mathrm{mK}$. The two last offsets cover the outer shells without contamination from the center.

Summing the molecular content of the two detected shells, we find $M\left(\mathrm{H}_{2}\right)=2.6 \times 10^{8} M_{\odot}$, see Table 2 .

The northern galaxy of the Arp 295 pair is a nearly edgeon spiral, which is strongly perturbed by the interaction and shows disordered shells and ripples. The observed spectra in Figs. 19 and 20 are essentially compatible with molecular gas in the disk, with some extension in the western shell (see Table 2). However, the emission does not extend far in the most western offset, so it is still marginal.

\section{Discussion}

The dynamical mechanism that forms shells through phase wrapping has been described in detail (Quinn 1984; Dupraz \& Combes 1986, 1987). Stars from a companion, liberated by tidal forces, oscillate in the potential of the primary galaxy, and a sharp edge forms as an outward-propagating wave at their apocenters. There could be radial oscillation in a prolate potential, forming alternate shells with zero velocity at their edge, or less radial ones with angular momentum, forming shells around the equatorial plane of an oblate potential, keeping some rotational velocity at their edge. Another mechanism is space wrapping,

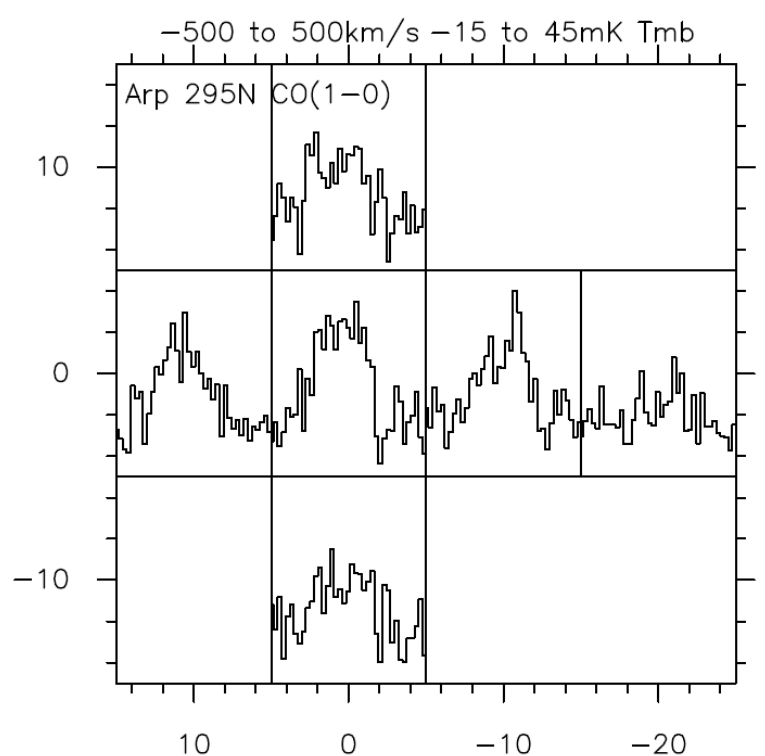

Fig. 19. CO(1-0) map of spectra in Arp 295N.

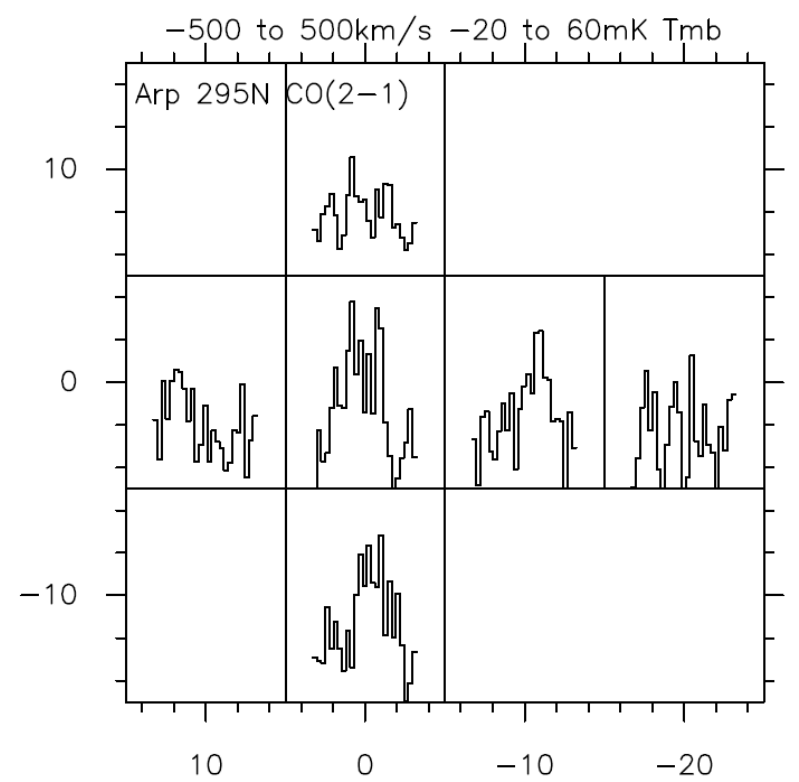

Fig. 20. $C O(2-1)$ map of spectra in Arp 295N.

where shells occur as ordinary loops in interactions and mergers, with higher velocities.

\subsection{Molecular clouds in shells}

Because gas is dissipative, it may appear surprising to find gas in shells. During radial oscillations, the diffuse gas is expected to fall into the center. Dense gas clouds do experience much less dissipation than diffuse HI gas. Moreover, numerical modeling of the shell formation (Combes \& Charmandaris 2000) using a realistic distribution of its stellar and gaseous component showed that the observed spatial distribution of gaseous and stellar shells can be easily reproduced within the standard models of the theory of shell formation. The detection of molecular gas associated with the shells in Centaurus A confirmed this model (Charmandaris et al. 2000). Using the Swedish-ESO Submillimetre Telescope (SEST), Charmandaris and collaborators 
detected a molecular gas of $4.3 \times 10^{7} M_{\odot}$ with the same kinematical characteristics as the HI shells.

The model predicts that a significant fraction of the mergerremnant dense molecular clouds has a dynamical behavior similar to that of the stellar component. Our detection of molecular clouds in shells through CO emission supports the model. With higher spatial resolution, it will be possible to determine the difference in position between the gas and stars in shells, and infer the loss of angular momentum during the encounter.

\subsection{CO velocity in shells}

CO emission in shells was clearly detected for three galaxies: Arp 10, NGC 3656, and NGC 3934. In the case of Arp 10, the inclination on the plane of the sky is small $\left(i=14^{\circ}\right.$ from Hyperleda), and the small velocity difference with respect to the systemic recession velocity for the gas in the shells is compatible with both phase and space wrapping. For the two other galaxies, the inclination is relatively large $\left(i=51^{\circ}\right.$ and $i=46^{\circ}$ for NGC 3656 and 3934, respectively), and the average velocity with respect to the systemic velocity of the shells is relatively low. However, the CO profiles in the shells are broad and show a two-horn shape even in both NGC 3656 and NGC 3934. This means that the $\mathrm{CO}$ beam that covers a large shell region, where the material is not only in radial orbits, but keeps a tangential velocity at the apocenter. In the line of sight toward the observer some material with positive and negative angular momentum with respect to the galaxy is projected.

This configuration is impossible to encounter with a disk in rotation. Two-horn profiles are only seen when the observation beam covers a wide region near the center, including both the approaching and receding sides. For an observation far from the center of the rotating disk, only one velocity peak (either blue or red) is expected. For shells, in contrast, the material from the companion is infalling on a nearly radial orbit, and part of the matter may arrive with a positive and part with a negative angular momentum with respect to the primary galaxy. In the shell region far from the center, it is possible to have the two velocity signs toward the same line of sight. The two-horn profile shape therefore supports the $\mathrm{CO}$ detection in shells. Confirmation of this scenario is required through observations with higher spatial resolution.

\subsection{Comparison with $\mathrm{HI}$ in shells}

HI has been mapped in Arp 230 (Schiminovich et al. 2013), NGC 474 (Rampazzo et al. 2006), Arp 10 (Charmandaris \& Appleton 1996), NGC 3032 (Oosterloo et al. 2010), NGC 3656 (Balcells et al. 2001), and Arp 295 (Hibbard \& van Gorkom 1996). In all these six galaxies, the HI is extended enough to cover the shells, and in NGC 3656, the HI is clearly seen associated with the southern stellar shell, with compatible kinematics. In most cases, however, the spatial resolution is not sufficient to clearly conclude that the gas provided by a small merging companion has crossed the system and oscillates in the shells. The only system with a clear presence of HI gas in shells is NGC 3656, which Balcells et al. (2001) compared to NGC 5128 (Centaurus A), where the first HI shells were discovered (Schiminovich et al. 1994). Given the orientation of the gas and dusty disk, which is perpendicular to the main stellar body, this shell system must have been formed through phase wrapping of material in radial orbits. $\mathrm{HI}$ and $\mathrm{CO}$ emission in the shells can only be explained by clumpy gas with a low-filling factor and with low dissipation (Combes \& Charmandaris 2000).

\section{Summary}

We have carried out $\mathrm{CO}(1-0)$ and $\mathrm{CO}(2-1)$ line observations of nine shell galaxies with the IRAM-30 m telescope. Molecular gas was detected in six of them, together with two of the companions. In three of the detected galaxies (Arp 230, NGC 3032, and Arp 205N), we cannot conclude that gas is detected in the shells; the detection is compatible with gas in the inner rotating disk. In the three remaining galaxies (Arp 10, NGC 3656, and NGC 3934), molecular gas is clearly detected in the shells, with a velocity close to systemic, and not in continuity with the inner rotating disk. The amount of molecular gas is estimated as $M\left(\mathrm{H}_{2}\right)=3.4 \times 10^{9} M_{\odot}$ in the shells outside the ring in Arp 10, $3.8 \times 10^{8} M_{\odot}$ in the southern shell of NGC 3656 , and $2.6 \times 10^{8} M_{\odot}$ in the two detected shells of NGC 3934.

The mechanism that formed these gaseous shells might be different. For two cases at least (Arp 10 and NGC 3656), the encounter with the small companion had a very small impact parameter, and the phase wrapping in a nearly radial orbit must have played the main role. The morphology of Arp 10 corresponds to an oblate system; this also holds for most shells in NGC 3656. However, the main shell might be only the end of the tidal tail. The gas of the companion of NGC 3656 has left a radial trace that is visible as a contrasted dust lane, and the main shell is found in continuity, indicating the companion orbit and that of the tidal debris. The main body of NGC 3934 is seen nearly edge-on in the sky, and the situation is less clear. Shells that are distributed symmetrically at about $40^{\circ}$ of the minor axis are clearly detected in molecular gas. The fact that gas can be detected in shells tends to support the clumpiness and low dissipative character of a large part of the interstellar medium. The line profiles in the shells reveal a two-horn shape, indicating both positive and negative angular momentum relative to the primary galaxy. This supports the $\mathrm{CO}$ detection in shells because it is impossible to explain a two-horn profile in the outer parts of the disk of a rotating galaxy. Material from the companion must have infallen in a nearly radial orbit, part of it with a positive and the other part a negative impact parameter. The material acquired a tangential velocity at the apocenter. The shells are then also phase wrapped. Follow-up with higher spatial resolution is required to test the relative position of gas and stellar shells to confirm the dynamical mechanism.

Acknowledgements. We warmly thank the referee for constructive comments and suggestions. This work is based on observations carried out with the IRAM $30 \mathrm{~m}$ telescope. IRAM is supported by INSU/CNRS (France), MPG (Germany) and IGN (Spain). This publication has made use of data products from the NASA/IPAC Extragalactic Database (NED). We acknowledge the usage of the HyperLeda database (http: //leda.univ-lyon1. fr).

\section{References}

Alatalo, K., Davis, T. A., Bureau, M., et al. 2013, MNRAS, 432, 1796 Appleton, P. N., \& Struck-Marcell, C. 1996, Fund. Cosmic Phys., 16, 111 Arp, H. 1966, ApJS, 14, 1

Balcells, M., \& Stanford, S. A. 1990, ApJ, 362, 443

Balcells, M., van Gorkom, J. H., Sancisi, R., \& del Burgo, C. 2001, AJ, 122, 1758

Barnes, J. E., \& Hernquist, L. 1992, ARA\&A, 30, 705

Bettoni, D., Galletta, G., Rampazzo, R., et al. 2011, A\&A, 534, A24

Bizyaev, D. V., Moiseev, A. V., \& Vorobyov, E. I. 2007, ApJ, 662, 304

Bolatto, A. D., Wolfire, M., \& Leroy, A. K. 2013, ARA\&A, 51, 207

Buson, L. M., Bertola, F., Bressan, A., Burstein, D., \& Cappellari, M. 2004, A\&A, 423, 965

Carter, D., Allen, D. A., \& Malin, D. F. 1982, Nature, 295, 126

Charmandaris, V., \& Appleton, P. N. 1996, ApJ, 460, 686

Charmandaris, V., Combes, F., \& van der Hulst, J. M. 2000, A\&A, 356, L1 
Chien, L.-H., Barnes, J. E., Kewley, L. J., \& Chambers, K. C. 2007, ApJ, 660 L105

Combes, F., \& Charmandaris, V. 2000, in Dynamics of Galaxies: from the Early Universe to the Present, eds. F. Combes, G. A. Mamon, \& V. Charmandaris, ASP Conf. Ser., 197, 339

Combes, F., Young, L. M., \& Bureau, M. 2007, MNRAS, 377, 1795

Cooper, A. P., Martínez-Delgado, D., Helly, J., et al. 2011, ApJ, 743, L21

Duc, P.-A., Cuillandre, J.-C., Karabal, E., et al. 2015, MNRAS, 446, 120

Dupraz, C., \& Combes, F. 1986, A\&A, 166, 53

Dupraz, C., \& Combes, F. 1987, A\&A, 185, L1

Galletta, G., Sage, L. J., \& Sparke, L. S. 1997, MNRAS, 284, 773

Hernquist, L., \& Quinn, P. J. 1988, ApJ, 331, 682

Hernquist, L., \& Quinn, P. J. 1989, ApJ, 342, 1

Hibbard, J. E., \& van Gorkom, J. H. 1996, AJ, 111, 655

Huchtmeier, W. K. 1982, A\&A, 110, 121

Kim, D.-W., Guhathakurta, P., van Gorkom, J. H., Jura, M., \& Knapp, G. R. 1988, ApJ, 330, 684

Lim, S., Peng, E. W., Duc, P.-A., et al. 2017, ApJ, 835, 123

Lucero, D. M., \& Young, L. M. 2013, AJ, 145, 56

McDermid, R. M., Emsellem, E., Shapiro, K. L., et al. 2006, MNRAS, 373, 906

McGaugh, S. S., \& Bothun, G. D. 1990, AJ, 100, 1073

Oosterloo, T., Morganti, R., Crocker, A., et al. 2010, MNRAS, 409, 500

Prieur, J.-L. 1988, ApJ, 326, 596

Quinn, P. J. 1984, ApJ, 279, 596

Rampazzo, R., Alexander, P., Carignan, C., et al. 2006, MNRAS, 368, 851

Rampazzo, R., Marino, A., Tantalo, R., et al. 2007, MNRAS, 381, 245

Renaud, F., Bournaud, F., \& Duc, P.-A. 2015, MNRAS, 446, 2038

Richter, O.-G., Sackett, P. D., \& Sparke, L. S. 1994, AJ, 107, 99

Sage, L. J., \& Wrobel, J. M. 1989, ApJ, 344, 204

Sansom, A. E., Hibbard, J. E., \& Schweizer, F. 2000, AJ, 120, 1946
Schiminovich, D., van Gorkom, J. H., van der Hulst, J. M., \& Kasow, S. 1994, ApJ, 423, L101

Schiminovich, D., van Gorkom, J. H., van der Hulst, J. M., \& Malin, D. F. 1995, ApJ, 444, L77

Schiminovich, D., van Gorkom, J., van der Hulst, T., Oosterloo, T., \& Wilkinson, A. 1997, in The Nature of Elliptical Galaxies; 2nd Stromlo Symposium, eds. M. Arnaboldi, G. S. Da Costa, \& P. Saha, ASP Conf. Ser., 116, 362

Schiminovich, D., van Gorkom, J. H., \& van der Hulst, J. M. 2013, AJ, 145, 34

Schweizer, F. 1990, Interactions in Our Time, ed. R. Wielen (Berlin, Heidelberg: Springer), 60

Schweizer, F., \& Seitzer, P. 1992, AJ, 104, 1039

Serra, P., Trager, S. C., Oosterloo, T. A., \& Morganti, R. 2008, A\&A, 483, 57

Sofue, Y., Wakamatsu, K.-I., Taniguchi, Y., \& Nakai, N. 1993, PASJ, 45, 43

Solomon, P. M., \& Vanden Bout, P. A. 2005, ARA\&A, 43, 677

Tateuchi, K., Konishi, M., Motohara, K., et al. 2015, ApJS, 217, 1

Toomre, A., \& Toomre, J. 1972, ApJ, 178, 623

Turnbull, A. J., Bridges, T. J., \& Carter, D. 1999, MNRAS, 307, 967

Ueda, J., Iono, D., Yun, M. S., et al. 2014, ApJS, 214, 1

van Driel, W., Arnaboldi, M., Combes, F., \& Sparke, L. S. 2000, A\&AS, 141, 385

van Gorkom, J. 2000, in Radio interferometry: The Saga and the Science, eds. D. G. Finley, \& W. M. Goss, 137

Weil, M. L., \& Hernquist, L. 1993, ApJ, 405, 142

Whitmore, B. C., Lucas, R. A., McElroy, D. B., et al. 1990, AJ, 100, 1489

Whitmore, B. C., Schweizer, F., Leitherer, C., Borne, K., \& Robert, C. 1993, AJ, 106, 1354

Wiklind, T., Combes, F., \& Henkel, C. 1995, A\&A, 297, 643

Young, L. M. 2002, AJ, 124, 788

Young, L. M., Bureau, M., Davis, T. A., et al. 2011, MNRAS, 414, 940 


\section{Appendix A: Molecular content of the companions}

The companion of the shell galaxy NGC 474 (Arp 227) is a late-type spiral galaxy, NGC 470, which is rich in $\mathrm{HI}$ and $\mathrm{CO}$ emission. It is likely that the HI extension that covers NGC 474 (see Fig. 3) has been accreted from this companion. The beams observed in NGC 470 are displayed in Fig. 2. The spectra maps in $\mathrm{CO}(1-0)$ and $\mathrm{CO}(2-1)$ are displayed in Figs. A.1 and A.2, respectively. Isocontours of the surface density and the velocity fields are plotted in Fig. A.3.

The other member of the pair in Arp 295 is an edge-on spiral galaxy that is rich in molecular gas. The pointings are displayed in an Hubble Space Telescope (HST) F814W image in Fig. A.4. CO spectra in Arp 295S are plotted in Figs. A.5 and A.6. The molecular content in each beam is detailed in Table A.1.

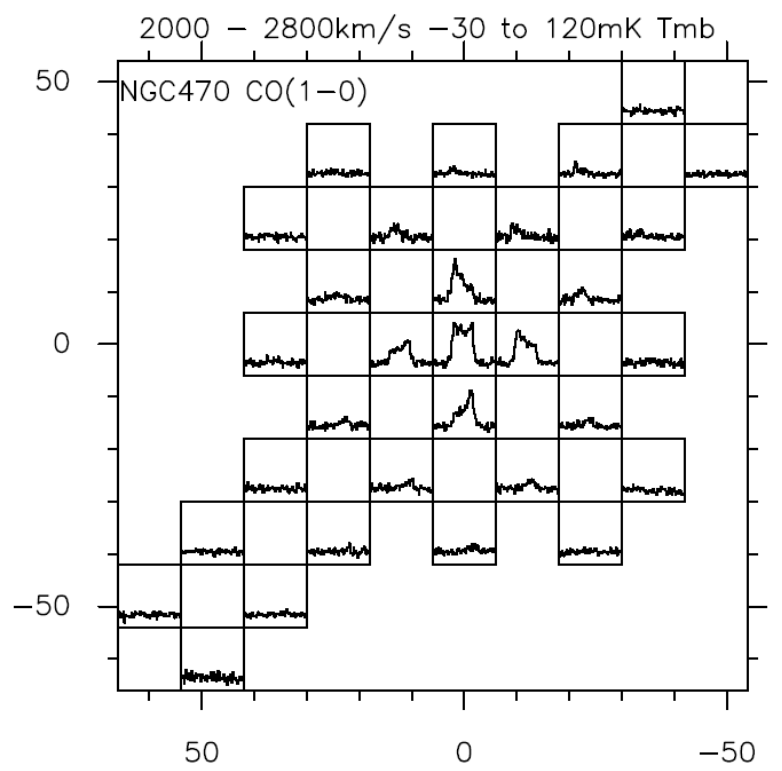

Fig. A.1. CO(1-0) map of spectra in NGC 470.

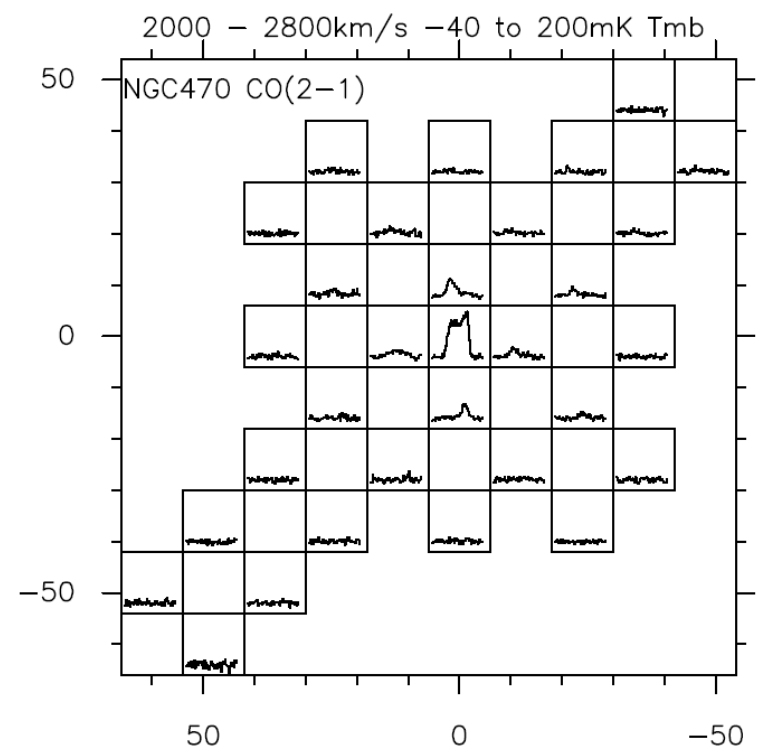

Fig. A.2. $\mathrm{CO}(2-1)$ map of spectra in NGC 470.

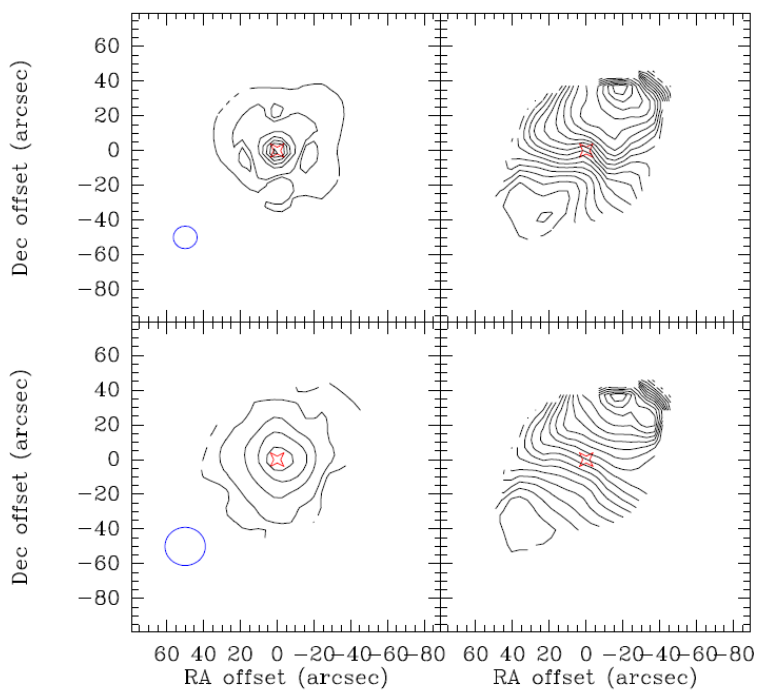

Fig. A.3. Isocontours of NGC 470 for $\mathrm{CO}(2-1)$ (upper panels) and $\mathrm{CO}(1-0)$ (lower panels), the right panels display the corresponding velocity fields. The isovels are spaced by $20 \mathrm{~km} \mathrm{~s}^{-1}$; they range from $2200 \mathrm{~km} \mathrm{~s}^{-1}$ (NW) to $2560 \mathrm{~km} \mathrm{~s}^{-1}$ (SE).

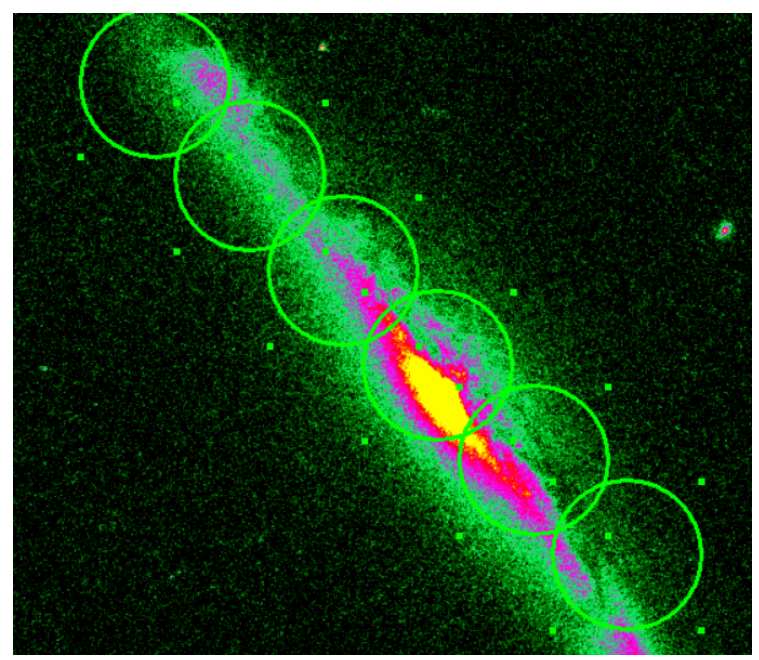

Fig. A.4. CO(2-1) 11" beams observed toward Arp 295S, overlaid on an HST F814W image. 
Table A.1. CO results for the companions.

\begin{tabular}{|c|c|c|c|c|c|c|c|c|c|}
\hline \multirow[b]{2}{*}{ Galaxy } & \multirow[b]{2}{*}{$\begin{array}{c}\text { Offsets } \\
\left({ }^{\prime \prime},{ }^{\prime \prime}\right)\end{array}$} & \multicolumn{3}{|c|}{$\mathrm{CO}(1-0)$} & \multicolumn{3}{|c|}{$\mathrm{CO}(2-1)$} & \multirow[b]{2}{*}{$\begin{array}{l}M\left(\mathrm{H}_{2}\right) \\
10^{8} M_{\odot}\end{array}$} & \multirow[b]{2}{*}{$\begin{array}{c}\Sigma\left(\mathrm{H}_{2}\right) \\
M_{\odot} \mathrm{pc}^{-2}\end{array}$} \\
\hline & & $\begin{array}{c}\text { Int-flux } \\
\text { Jy km s }^{-1}\end{array}$ & $\begin{array}{c}V \\
\mathrm{~km} \mathrm{~s}^{-1}\end{array}$ & $\begin{array}{c}\Delta V \\
\mathrm{~km} \mathrm{~s}^{-1}\end{array}$ & $\begin{array}{c}\text { Int-flux } \\
\text { Jy km s }^{-1}\end{array}$ & $\begin{array}{c}V \\
\mathrm{~km} \mathrm{~s}^{-1}\end{array}$ & $\begin{array}{c}\Delta V \\
\mathrm{~km} \mathrm{~s}^{-1}\end{array}$ & & \\
\hline NGC 470 & $\begin{array}{c}(0,0) \\
\text { see }\end{array}$ & $\begin{array}{c}126 \pm 2 \\
31 \mathrm{pt}\end{array}$ & $\begin{array}{c}66 \pm 2 \\
\text { map }\end{array}$ & $252 \pm 4$ & $216 \pm 2$ & $88 \pm 1$ & $246 \pm 2$ & $14 \pm 0.2$ & 151 \\
\hline$\overline{\text { ARP 295S }}$ & $\begin{array}{c}(0,0) \\
(-7,-7) \\
(-14,-14) \\
(7,7) \\
(14,14) \\
(21,21)\end{array}$ & $\begin{array}{c}35.3 \pm 3 \\
39 \pm 3.5 \\
19 \pm 1.6 \\
22 \pm 2 \\
9.2 \pm 1.1 \\
3.6 \pm 0.7\end{array}$ & $\begin{array}{c}82 \pm 22 \\
-82 \pm 16 \\
-173 \pm 10 \\
-192 \pm 12 \\
256 \pm 11 \\
272 \pm 7\end{array}$ & $\begin{array}{c}512 \pm 48 \\
355 \pm 40 \\
248 \pm 25 \\
238 \pm 27 \\
169 \pm 25 \\
71 \pm 17\end{array}$ & $\begin{array}{c}<4 \\
27.1 \pm 2.5 \\
6.4 \pm 1.3 \\
6.5 \pm 3 \\
<2.5 \\
<2.5\end{array}$ & $\begin{array}{c}- \\
-74 \pm 11 \\
-150 \pm 7 \\
239 \pm 38 \\
- \\
-\end{array}$ & $\begin{array}{c}- \\
256 \pm 24 \\
80 \pm 23 \\
176 \pm 96 \\
- \\
-\end{array}$ & $\begin{array}{c}32.5 \pm 3 \\
36 \pm 3 \\
17 \pm 1.5 \\
20.3 \pm 2 \\
8.5 \pm 1 \\
3.3 \pm 0.6\end{array}$ & $\begin{array}{c}42 \\
47 \\
22 \\
26 \\
11 \\
4\end{array}$ \\
\hline
\end{tabular}

Notes. Integrated flux, velocity, and FWHM obtained through Gaussian fits. Velocities are counted from the central redshifts indicated in Table 1 for the companion galaxy. The $\mathrm{H}_{2}$ masses are estimated within the $\mathrm{CO}(1-0)$ beam of 23", with the standard conversion factor of the Milky Way $\alpha=4.36 M_{\odot}\left(\mathrm{K} \mathrm{km} \mathrm{s}^{-1} \mathrm{pc}^{2}\right)^{-1}$. Upper limits are given at $3 \sigma$, assuming an FWHM of $300 \mathrm{~km} \mathrm{~s}^{-1}$.

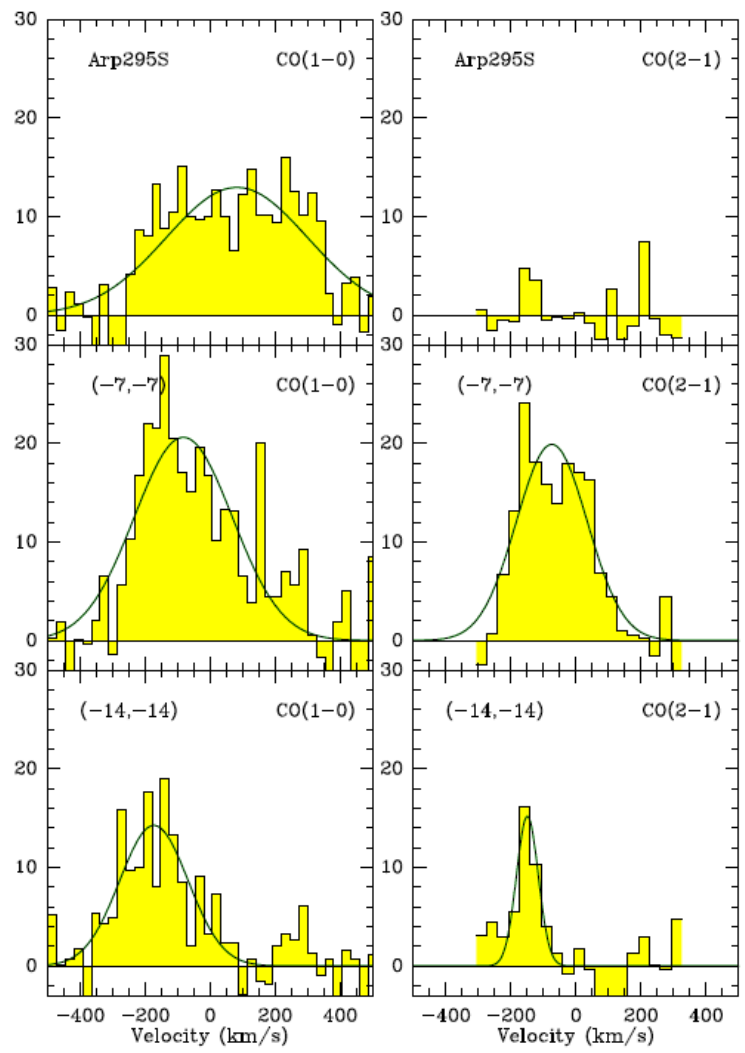

Fig. A.5. CO spectra in Arp 295S, central position and offsets (in arc$\mathrm{sec})$. The vertical axis is the main-beam temperature in $\mathrm{mK}$.

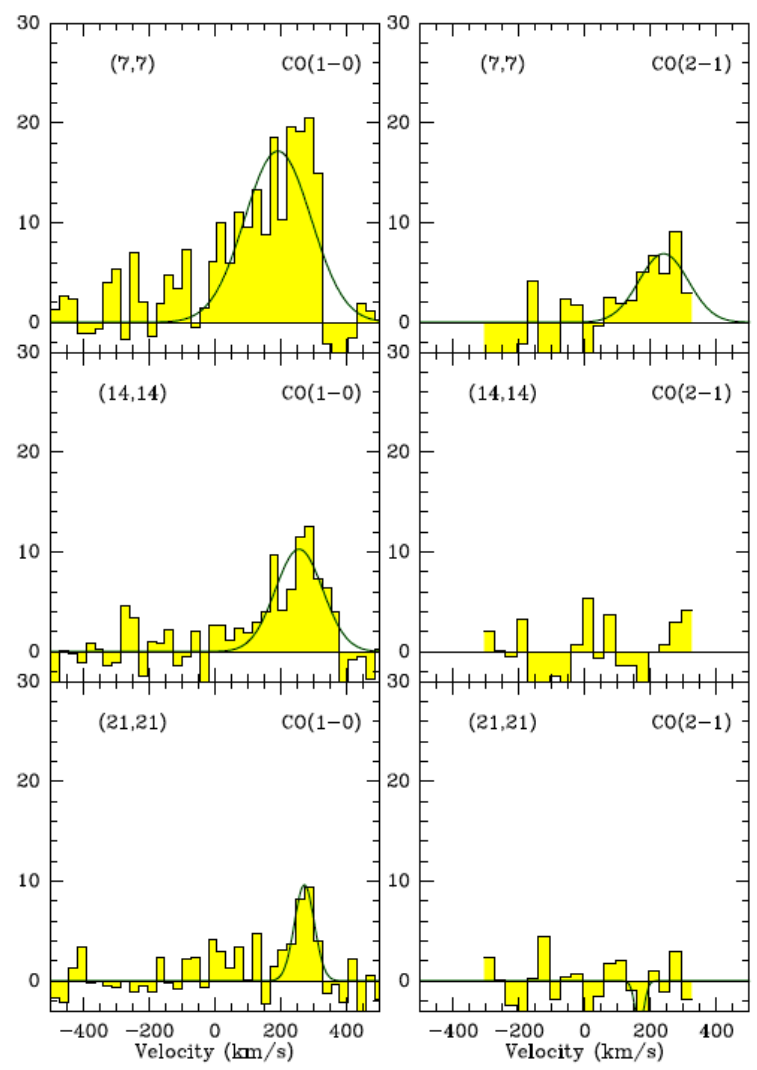

Fig. A.6. CO spectra in Arp 295S (following), northern offsets (in arc$\mathrm{sec})$. The vertical axis is the main-beam temperature in $\mathrm{mK}$. 